\title{
Spin System Assignment of ortho-Phenylene Ethynylene Oligomers
}

\author{
Morris M. Slutsky, Ticora V. Jones, Gregory N. Tew* \\ Contribution from the Polymer Science \& Engineering Department, University of Massachusetts, \\ Amherst, Massachusetts 01003
}

tew@mail.pse.umass.edu

\section{SUPPLEMENTAL INFORMATION}

Experimental Section

$\mathrm{S} 2-\mathrm{S} 3$

Synthetic Procedures.

Summary Tables of HMBC and COSY Interactions.

S12-S14

HPLC and NMR Confirmation of Es ${ }_{6}$ Purity.

S15

HMBC for Es ${ }_{6}$ Ester-Carbon Aromatic-Proton Region.

S16

COSY Data, Aromatic Proton Regions.

S17-S19

HMBC for Es ${ }_{4-6}, \alpha 1-\alpha 2-C a r b o n$ Aromatic-Proton Region.

S20-S22

HMBC for Es4-6 Acetylene-Carbon Aromatic-Proton Region.

S23-S25

Details and Discussion for Assignment of Long-Range HMBC Peaks.

S26-S30 


\section{EXPERIMENTAL}

\section{SAMPLE PREPARATION}

The synthesis of Es 4 has been previously reported. ${ }^{25} \mathrm{Es}_{5}$ and $\mathrm{Es}_{6}$ oligomers have been synthesized by similar techniques. NMR samples were prepared in saturated concentration in $\mathrm{CD}_{3} \mathrm{CN}$, a solvent that provides good dispersion of signals and high solubility $(\approx 50 \mathrm{mg} / \mathrm{mL})$. For purposes of verifying purity of $\mathrm{Es}_{6}$, a $1.25 \mathrm{mM}$ sample in $\mathrm{CD}_{3} \mathrm{CN}$ was also prepared.

\section{HPLC}

Purity of Es 4 and $\mathrm{Es}_{5}$ was apparent by NMR spectroscopy, but the spectrum of Es 6 at high concentration showed extra peaks which we have attributed to aggregation as previously discussed. Purity of $\mathrm{Es}_{6}$ was verified by chromatography on an analytical $4.5 \times 150 \mathrm{~mm}$ ODS $5 \mu \mathrm{m}$ silica column, eluted with a gradient of $50 \%->100 \%$ acetonitrile $/ \mathrm{H}_{2} \mathrm{O}$ at $1.0 \mathrm{~mL} / \mathrm{min}$, monitored by absorbance at 254 nm. A photodiode array detector was used. HPLC indicated purity of $95.6 \%$.

\section{NMR SPECTROSCOPY}

Spectra were recorded with a $600 \mathrm{MHz}$ spectrometer by means of a TXI probe with Z-gradient capabilities. The temperature was maintained at $300 \mathrm{~K}$ for all acquisitions, with the exception of COSY of Es 6 performed at $305 \mathrm{~K}$. One-dimensional ${ }^{1} \mathrm{H}$ and ${ }^{13} \mathrm{C}$ spectroscopy, gradient correlated J-coupled correlation spectroscopy (GCOSY), and heteronuclear multiple bond correlation (HMBC) were used for assignment of $\mathrm{Es}_{4}, \mathrm{Es}_{5}$, and $\mathrm{Es}_{6}$ oligomers.

\section{STRUCTURE DETERMINATION}

COSY spectra of $\mathrm{Es}_{4}, \mathrm{Es}_{5}$, and $\mathrm{Es}_{6}$ were taken using a gradient-selective pulse sequence. Sweep width was set to the aromatic ${ }^{1} \mathrm{H}$ region, typically $1200 \mathrm{~Hz}$. A relaxation delay of 2 seconds was used, with 12-16 scans and 128 increments. Processing was performed with $1 \mathrm{~K}$ x $1 \mathrm{~K}$ data points, using sinebell multiplication in both dimensions. HMBC spectra of Es 4 and $\mathrm{Es}_{6}$ were performed with the standard 
HMBC pulse program. The number of scans taken was 56, with 512 increments. Spectra covering the entire ${ }^{1} \mathrm{H}$ and ${ }^{13} \mathrm{C}$ region were initially taken in order to locate the $\mathrm{Si}\left(\mathrm{CH}_{3}\right)_{3}$ peaks. In order to obtain better resolution for $\mathrm{Es}_{6}$, the sweep width was reduced to fit the aromatic ${ }^{1} \mathrm{H}$ region and the ${ }^{13} \mathrm{C}$ ester, aromatic, and acetylene regions, to $673 \mathrm{~Hz}$ in the ${ }^{1} \mathrm{H}$ dimension and $15436 \mathrm{~Hz}$ in the ${ }^{13} \mathrm{C}$ dimension, with 32 scans and 1000 increments. HMBC spectra of Es 5 were performed with the use of a doublequantum filtered gradient HMBC program with long-range optimization and low-pass J-filtering. Spectra covering the entire ${ }^{1} \mathrm{H}$ and ${ }^{13} \mathrm{C}$ region were initially taken to locate the $\mathrm{Si}\left(\mathrm{CH}_{3}\right)_{3}$ peak. In order to obtain better resolution and signal/noise ratio, the sweep width was reduced to fit the aromatic ${ }^{1} \mathrm{H}$ region and the ${ }^{13} \mathrm{C}$ ester, aromatic, and acetylene regions, to $673 \mathrm{~Hz}$ in the ${ }^{1} \mathrm{H}$ dimension and $13661 \mathrm{~Hz}$ in the ${ }^{13} \mathrm{C}$ dimension, with 56 scans and 1100 increments. An initial experiment was made with an evolution delay of $60 \mathrm{~ms}$, and was used to identify short-range HMBC couplings (not crossing carboncarbon triple bonds). A further experiment was made with an evolution delay of $150 \mathrm{~ms}$, in order to identify longer-range HMBC couplings across carbon-carbon triple bonds. 
Measurements. ${ }^{1} \mathrm{H}$, and ${ }^{13} \mathrm{C}$ NMR spectra for general analysis were obtained with a $400 \mathrm{MHz}$ NMR spectrometer. Mass spectral data were obtained at the University of Massachusetts Amherst mass spectroscopy facility, which is supported in part by the National Science Foundation.

Materials. Reagent grade tetrahydrofuran (THF) was distilled under nitrogen from sodium benzophenone. All other solvents were used as received. Trans dichlorobis (triphenylphosphine) palladium $\left(\mathrm{Pd}\left(\mathrm{P}_{3}\right)_{2} \mathrm{Cl}_{2}\right)$ was purchased from Strem Chemical. Tri-ethylene glycol monomethyl ether was purchased from Aldrich and used after solvation in dichloromethane, and passing through a pipette of silica and subsequent evaporation. The compound was then evaporated and dried under vacuum. Trimethylsilyl acetylene was purchased from GFS chemicals. All other reagents were used as received.

Purification. All column chromatography was performed on an automated flash chromatography system using the column sizes and solvent gradients as indicated.

Abbreviations used: DCM (dichloromethane), TBAF (Tetra butyl ammonium fluoride), EtOAc (ethyl acetate), TEA (triethylamine), TMS (trimethylsilyl).

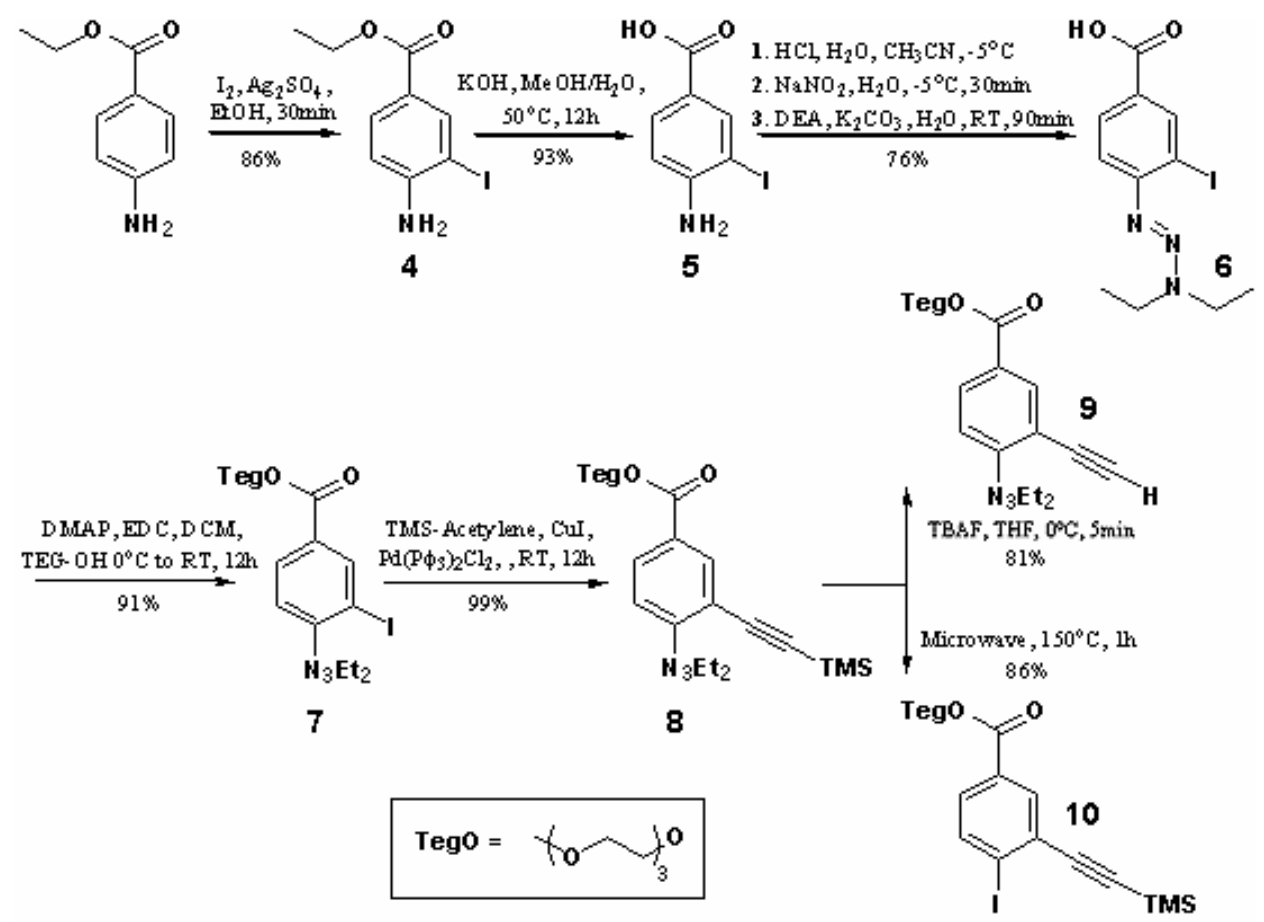

Figure S1 Synthetic scheme for Teg-ester monomer. 

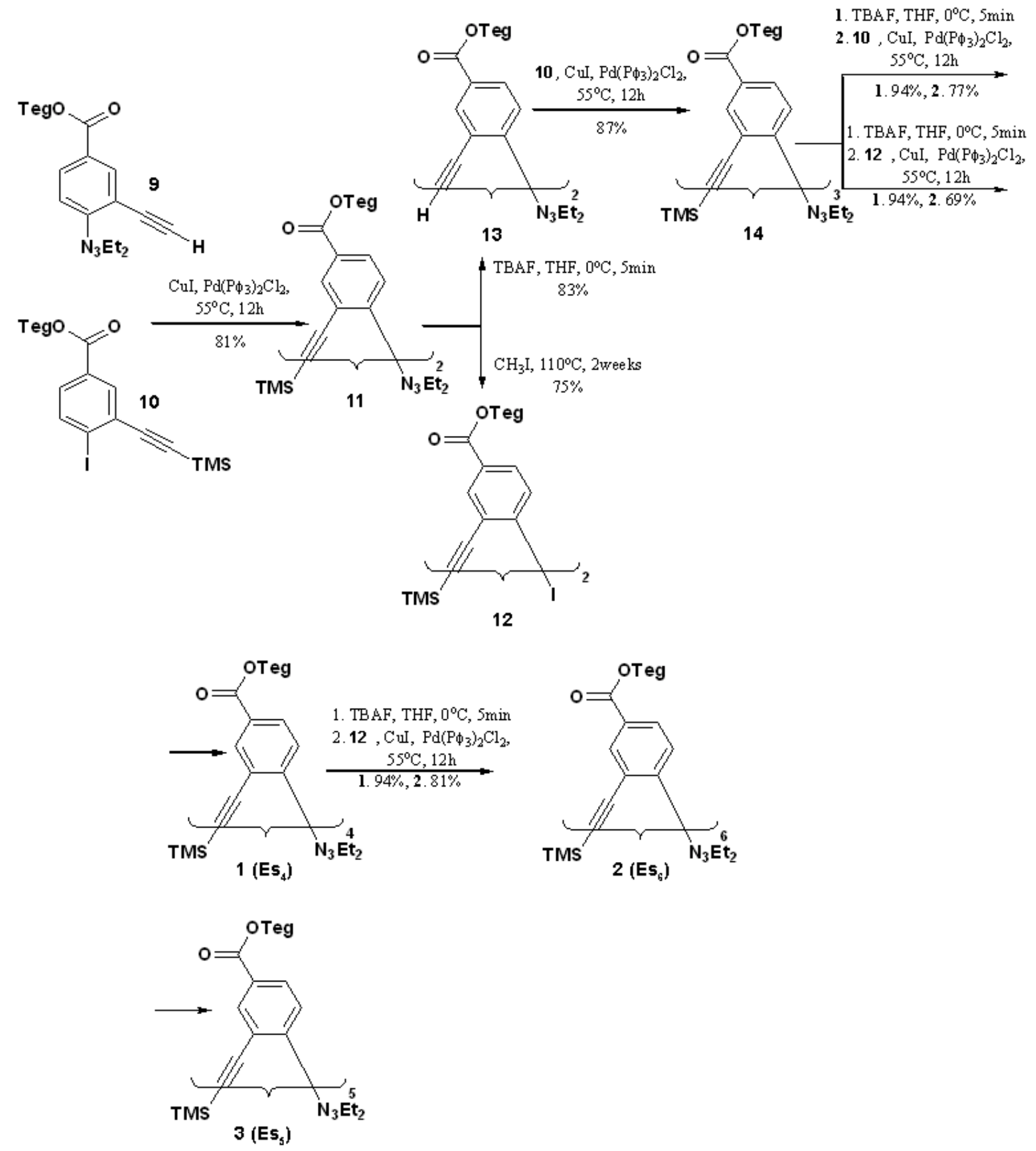

Figure S2 Synthetic scheme for Teg-ester monomer.

General TMS Deprotection Procedure (TBAF). One equivalent of the TMS protected compound was dissolved in dry THF and cooled to $0^{\circ} \mathrm{C}$ in a round bottom flask with stirbar. 1.2 equivalents of TBAF in $1 \mathrm{M}$ THF solution with $5 \% \mathrm{H}_{2} \mathrm{O}$ content were added, and the reaction was stirred for 5 minutes. Enough hexane was then added to bring the reaction to a 1:1 THF/hexane ratio, precipitating most excess TBAF and t-butyl ammonium hydroxide, and the reaction was stirred for an additional 10 minutes. The reaction mixture was injected directly, without evaporation, onto either a silica-packed pipette or a flash chromatography column for purification. 
General Triazene Activation Procedure. This procedure was performed by microwave synthesis in Biotage 2-5 mL vials. Each vial was filled with $250 \mathrm{mg}$ of triazene protected compound 0.05 eq of $\mathrm{I}_{2}$ and of $\mathrm{MeI} \approx 130 \mathrm{eq}$, a stirbar was added, and a septum crimped on. Microwave heat was applied to each tube, at a temperature of $150^{\circ} \mathrm{C}$ for a time of one hour. After all reactions had completed, the tubes were opened, combined, filtered through a Celite pad and washed with ethyl ether, and evaporated under a $\mathrm{N}_{2}$ stream, and purified by flash chromatography.

General Sonogashira Coupling Procedure. A schlenk flask with stirbar was flame dried under vacuum and backfilled with $\mathrm{N}_{2}$ three times. To this flask were added 0.05-0.1 equivalents (based on the acetylene compound) of $\mathrm{Pd}\left(\mathrm{P}_{3}\right)_{2} \mathrm{Cl}_{2}$ and 0.1-0.2 equivalents of $\mathrm{CuI}$. The 1-1.1 equivalents of the acetylene compound to 1 equivalent iodide were dissolved in separate flasks in TEA and transferred via syringe to the schlenk flask under $\mathrm{N}_{2}$. The schlenk flask was gently degassed for 30 seconds then backfilled with $\mathrm{N}_{2}$. The flask sealed and placed in an oil bath at $55^{\circ} \mathrm{C}$ for at least $6-18$ hours and checked by TLC for completeness. A precipitate should form. Once done, the reaction solution was diluted with ether, filtered through a pad of Celite and concentrated. The residue was then purified using Silica flash chromatography using the solvents indicated.

4-Amino-3-iodo-benzoic acid ethyl ester (4) $13.8 \mathrm{~g}$ of $\mathrm{I}_{2}(54.5 \mu \mathrm{mol}, 1.0 \mathrm{~mol}$ eq.), and $17.0 \mathrm{~g}$ of $\mathrm{Ag}_{2} \mathrm{SO}_{4}(54.5 \mu \mathrm{mol}, 1.0 \mathrm{~mol}$ eq.) were added to $300 \mathrm{~mL}$ of $95 \% \mathrm{EtOH}$ with rapid stirring. $9.00 \mathrm{~g}$ of 4 -amino benzoic acid ethyl ester $(54.5 \mu \mathrm{mol}, 1.0 \mathrm{~mol}$ eq.) was dissolved in another $100 \mathrm{~mL}$ EtOH and added to the reaction, which was stirred at room temperature for 30 minutes. Mixture was filtered through frit to remove salts and the EtOH was removed by rotary evaporation. Residue was partitioned between $300 \mathrm{~mL}$

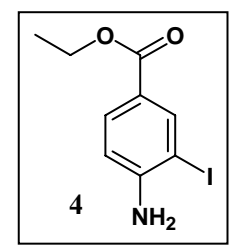

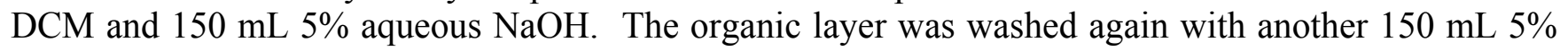
aqueous $\mathrm{NaOH}$, followed by washes with $2150 \mathrm{~mL}$ portions of $5 \%$ aqueous $\mathrm{Na}_{2} \mathrm{SO}_{3}$, dried over $\mathrm{MgSO}_{4}$, and evaporated. After purification by flash chromatography (85->100\% DCM: Hexanes) a tan solid was obtained $(13.6 \mathrm{~g}, 86 \%) .{ }^{1} \mathrm{H}$ NMR $\left(\mathrm{DMSO}_{\mathrm{d}}\right)$ ): $\delta 8.102(\mathrm{~d}, 1 \mathrm{H}$, phenyl H, J = 2.0), $7.652(\mathrm{dd}$, $1 \mathrm{H}$, phenyl $\left.\mathrm{H}, \mathrm{J}_{1}=2.0, \mathrm{~J}_{2}=8.4\right), 6.743(\mathrm{~d}, 1 \mathrm{H}$, phenyl $\mathrm{H}, \mathrm{J}=8.4), 6.065\left(\mathrm{~s}, 2 \mathrm{H}, \mathrm{NH}_{2}\right), 4.207(\mathrm{q}, 2 \mathrm{H}, \mathrm{J}=$ 7.2, $\left.\mathrm{CH}_{2}\right), 1.267\left(\mathrm{t}, 3 \mathrm{H}, \mathrm{J}=7.2, \mathrm{CH}_{3}\right) \mathrm{ppm} .{ }^{13} \mathrm{C} \mathrm{NMR}\left(\mathrm{DMSO}-\mathrm{d}_{6}\right): \delta 164.53,152.94,140.25,130.50$, $118.45,112.92,81.07,60.00,14.29 \mathrm{ppm}$. MS $\mathrm{m} / \mathrm{z}=291\left(\mathrm{~m}+\mathrm{H}^{+}\right)$.

4-Amino-3-iodo-benzoic acid (5) A solution of $13.0 \mathrm{~g} \mathrm{KOH} \mathrm{(232} \mathrm{mmol} \mathrm{,} 5.0 \mathrm{eq})$ in 700 $\mathrm{mL} 3: 1 \mathrm{MeOH} / \mathrm{H}_{2} \mathrm{O}$ was made, and $13.6 \mathrm{~g}$ of 4 (46.7 mmol, $\left.1.0 \mathrm{eq}\right)$ was added. The mixture was stirred at $50^{\circ} \mathrm{C}$ overnight. Methanol was removed by rotary evaporation, and the mixture was brought to $\mathrm{pH} 3.0$ by careful addition of concentrated $\mathrm{H}_{2} \mathrm{SO}_{4}$. The product was obtained as a white precipitate and was isolated by filtration $(11.42 \mathrm{~g}, 93 \%)$.

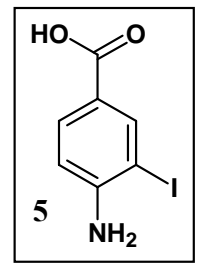

${ }^{1} \mathrm{H}$ NMR $\left(\right.$ DMSO-d $\left._{6}\right): \delta 8.091\left(\mathrm{~d}, 1 \mathrm{H}\right.$, phenyl H, J = 2.0), $7.631\left(\mathrm{dd}, 1 \mathrm{H}\right.$, phenyl H, $\mathrm{J}_{1}=$ $\left.2.0 \mathrm{~J}_{2}=8.4\right), 6.731(\mathrm{~d}, 1 \mathrm{H}$, phenyl $\mathrm{H}, \mathrm{J}=8.4), 5.97\left(\mathrm{br}, 2 \mathrm{H}, \mathrm{NH}_{2}\right){ }^{13} \mathrm{C}$ NMR $\left(\mathrm{DMSO}-\mathrm{d}_{6}\right)$ : $\delta 166.14,152.63,140.54,130.71,119.40,112.89,81.10 \mathrm{ppm} . \mathrm{MS} m / \mathrm{z}=263\left(\mathrm{~m}+\mathrm{H}^{+}\right)$.

$\boldsymbol{N}, \boldsymbol{N}$-Diethyl- $\boldsymbol{N}^{\prime}(2-I o d o-4-b e n z o i c$ acid) triazene (6) A solution of $5.81 \mathrm{~g}$ of 5 (22.1 mmol, $1.0 \mathrm{eq}$ ) in $380 \mathrm{~mL}$ of acetonitrile, $80 \mathrm{~mL}$ of water, and $11 \mathrm{~mL}$ concentrated $\mathrm{HCl}$

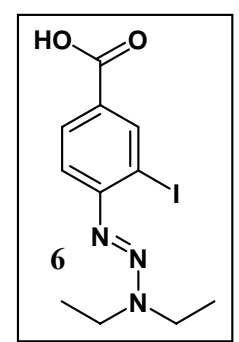
in a $1000 \mathrm{~mL}$ round bottom flask with stirbar was covered with aluminum foil to protect contents from light, and cooled in $\mathrm{a}-5^{\circ} \mathrm{C}$ ice/acetone bath. A solution of $3.35 \mathrm{~g} \mathrm{NaNO}_{2}$ (48.6 mmol, $\left.2.2 \mathrm{eq}\right)$ in $50 \mathrm{~mL}$ 
of ice/water was slowly added through an addition funnel. After the addition was complete the mixture was cannulated into a $2000 \mathrm{~mL}$ round bottom flask with stirbar containing a solution of $4.85 \mathrm{~g}$ diethylamine $(6.93 \mathrm{~mL}, 66.3 \mathrm{mmol}, 3.0 \mathrm{eq})$ and $9.16 \mathrm{~g} \mathrm{~K}_{2} \mathrm{CO}_{3}(66.3 \mathrm{mmol}, 3.0 \mathrm{eq})$ in $80 \mathrm{~mL}$ water in a $5^{\circ} \mathrm{C}$ ice/acetone bath. Reaction was stirred for 90 minutes, and then allowed to warm up to room temperature. Concentrated $\mathrm{HCl}$ was added to bring $\mathrm{pH}$ to 4.5 , and mixture was then extracted with 3 $300 \mathrm{~mL}$ portions of ethyl ether. The extract was dried over $\mathrm{MgSO}_{4}$ and evaporated to obtain crude product as an orange/red solid. After purification by flash chromatography $(0->15 \%$ EtOAc/DCM) a light yellow solid was obtained $(5.83 \mathrm{~g}, 76 \%) . \delta 8.591(\mathrm{~d}, 1 \mathrm{H}$, phenyl $\mathrm{H}, \mathrm{J}=2), 8.003(\mathrm{dd}, 1 \mathrm{H}$, phenyl $\mathrm{H}$, $\left.\mathrm{J}_{1}=2 \mathrm{~J}_{2}=8.4\right), 7.423(\mathrm{~d}, 1 \mathrm{H}$, phenyl $\mathrm{H}, \mathrm{J}=8.4), 3.829-3.882\left(\mathrm{~m}, 4 \mathrm{H}, \mathrm{CH}_{2}\right), 1.313-1.397\left(\mathrm{~m}, 6 \mathrm{H}, \mathrm{CH}_{2}\right)$ ppm. ${ }^{13} \mathrm{C} \mathrm{NMR}\left(\mathrm{CDCl}_{3}\right): \delta 171.05,154.74,141.58,130.87,126.66,116.82,95.92,49.87,42.93,14.59$, 11.02. $\mathrm{MS} m / \mathrm{z}=347\left(\mathrm{~m}+\mathrm{H}^{+}\right)$.

$N, N$-Diethyl- $N^{\prime} \quad$ 2-Iodo-4-benzoic acid 2-[2-(2-methoxy-ethoxy)-ethoxy]-ethyl ester\} triazene (7) $7.03 \mathrm{~g}$ of 6 (20.3 mmol, $1.05 \mathrm{eq})$ was dissolved in $200 \mathrm{~mL}$ of dry DCM to which $3.79 \mathrm{~g}$ of dimethylaminopyridine $(30.9 \mathrm{mmol}, 1.6 \mathrm{eq})$ was added. Mixture was cooled to $0^{\circ} \mathrm{C}$, and $5.92 \mathrm{~g}$ of 1-ethyl-3-(3-dimethylaminopropyl)carbodiimide (EDC) (30.9 mmol, $1.6 \mathrm{eq})$ in $100 \mathrm{~mL}$ dry DCM was added. After 20 minutes, $3.17 \mathrm{~g}$ triethylene glycol monomethyl ether (19.3 mmol, $1.0 \mathrm{eq})$ was added in $50 \mathrm{~mL}$ DCM. The reaction was stirred overnight, during which time the ice was allowed to melt. The mixture was evaporated and the residue partitioned between 60

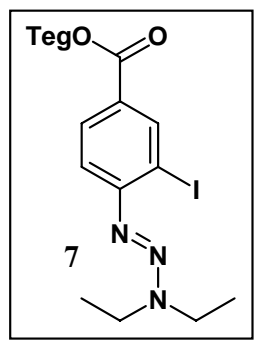
$\mathrm{mL}$ water and $2800 \mathrm{~mL}$ portions of EtOAc. The organic layer was washed with brine, dried over $\mathrm{MgSO}_{4}$, and evaporated to give crude product. Purification by flash chromatography in $30->50 \%$ EtOAc/Hexanes gave a light yellow oil $(8.70 \mathrm{~g}, 91 \%) .{ }^{1} \mathrm{H} \mathrm{NMR}\left(\mathrm{CDCl}_{3}\right): \delta 8.490(\mathrm{~d}, 1 \mathrm{H}$, phenyl $\mathrm{H}, \mathrm{J}=$ 1.6), $7.921\left(\mathrm{dd}, 1 \mathrm{H}\right.$, phenyl $\left.\mathrm{H}, \mathrm{J}_{1}=1.6 \mathrm{~J}_{2}=8.4\right), 7.361(\mathrm{~d}, 1 \mathrm{H}$, phenyl $\mathrm{H}, \mathrm{J}=8.4), 4.430(\mathrm{~m}, 2 \mathrm{H}$, $\left.\mathrm{CO}_{2} \mathrm{CH}_{2}\right), 3.80\left(\mathrm{~m}, 6 \mathrm{H}, \mathrm{CH}_{2}\right), 3.65\left(\mathrm{~m}, 6 \mathrm{H}, \mathrm{CH}_{2}\right), 3.52\left(\mathrm{~m}, 2 \mathrm{H}, \mathrm{CH}_{2}\right), 3.341\left(\mathrm{~s}, 3 \mathrm{H}, \mathrm{OCH}_{3}\right), 1.309(\mathrm{~m}$, $\left.6 \mathrm{H}, \mathrm{CH}_{3}\right) \mathrm{ppm} .{ }^{13} \mathrm{C} \mathrm{NMR}\left(\mathrm{CDCl}_{3}\right): \delta 165.33,153.92,140.71,130.24,127.47,116.61,95.79,71.95$, $70.72,70.64,70.62,69.23,64.15,59.07,49.66,42.70 \mathrm{ppm} . \mathrm{MS} \mathrm{m} / \mathrm{z}=493\left(\mathrm{~m}+\mathrm{H}^{+}\right)$.

$N, N$-Diethyl- $N$ ' \{2- trimethylsilanylethynyl 4-benzoic acid 2-[2-(2-methoxyethoxy)-ethoxyl-ethyl ester\} triazene (8) The general Sonogashira coupling procedure described above was used to prepare this compound. $8.70 \mathrm{~g}$ of 7 (17.6 mmol, $1.0 \mathrm{eq}), 440 \mathrm{mg}$ of $\mathrm{Pd}\left(\mathrm{P}_{3}\right)_{2} \mathrm{Cl}_{2}(0.63 \mathrm{mmol}, 0.04 \mathrm{eq})$, and $34 \mathrm{mg} \mathrm{CuI}$ $(0.176 \mathrm{mmol}, 0.01 \mathrm{eq})$ were combined in a $330 \mathrm{~mL}$ schlenk flask with $180 \mathrm{~mL}$ TEA. TMS acetylene $(3.73 \mathrm{~mL} / 2.60 \mathrm{~g}, 26.5 \mathrm{mmol}, 1.5 \mathrm{eq})$ was added to the solution. Reaction was stirred overnight at room temperature. After completion, the reaction solution was filtered through Celite with ether to wash, evaporated,

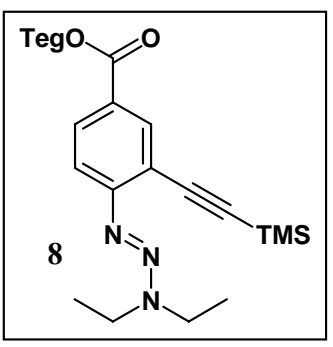
and purified with flash chromatography in 20\%- $>40 \% \mathrm{EtOAc} /$ hexanes to give a light yellow oil $(8.11 \mathrm{~g}$, 99\%). ${ }^{1} \mathrm{H} \mathrm{NMR}\left(\mathrm{CDCl}_{3}\right): \delta 8.162(\mathrm{~d}, 1 \mathrm{H}$, phenyl $\mathrm{H}, \mathrm{J}=2.0), 7.902\left(\mathrm{dd}, 1 \mathrm{H}\right.$, phenyl $\left.\mathrm{H}, \mathrm{J}_{1}=2.0 \mathrm{~J}_{2}=8.8\right)$, $7.441(\mathrm{~d}, 1 \mathrm{H}$, phenyl $\mathrm{H}, \mathrm{J}=8.8), 4.459\left(\mathrm{~m}, 2 \mathrm{H}, \mathrm{CO}_{2} \mathrm{CH}_{2}\right), 3.84\left(\mathrm{~m}, 6 \mathrm{H}, \mathrm{CH}_{2}\right), 3.69\left(\mathrm{~m}, 6 \mathrm{H}, \mathrm{CH}_{2}\right), 3.53$ $\left(\mathrm{m}, 2 \mathrm{H}, \mathrm{CH}_{2}\right), 3.365\left(\mathrm{~s}, 3 \mathrm{H}, \mathrm{OCH}_{3}\right), 1.31\left(\mathrm{~m}, 6 \mathrm{H}, \mathrm{CH}_{3}\right), 0.248\left(\mathrm{~s}, 9 \mathrm{H}, \mathrm{Si}\left(\mathrm{CH}_{3}\right)_{3}\right) \mathrm{ppm} .{ }^{13} \mathrm{C}$ $\operatorname{NMR}\left(\mathrm{CDCl}_{3}\right): \delta 166.15,156.24,135.31,130.58,125.94,118.03,116.53,102.64,98.83,72.07,70.83$, $70.79,70.75,69.41,64.15,59.19,49.69,42.40,14.62,11.09,0.16 \mathrm{ppm} \mathrm{MS} \mathrm{m/z}=464\left(\mathrm{~m}+\mathrm{H}^{+}\right)$. 


\section{$N, N$-Diethyl- $N$ ' $\{2$ - ethynyl 4-benzoic acid 2-[2-(2-methoxy-ethoxy)-ethoxy]- ethyl ester\} triazene (9)}

The general TMS deprotection procedure, listed above, was used to prepare this compound. $640 \mathrm{mg}$ of 8 (1.40 mmol, $1.0 \mathrm{eq})$ was dissolved in $5.4 \mathrm{~mL} \mathrm{THF}$, and the solution was cooled to $0^{\circ} \mathrm{C}$. $1.67 \mathrm{~mL}$ of $1 \mathrm{M} \mathrm{TBAF} / \mathrm{THF}$ with $5 \%$ water content was added to the mixture. After 5 minutes stirring, $5.4 \mathrm{~mL}$ hexane was added and the reaction stirred another 10 minutes. Purification was performed by filtration of the reaction mixture through silica gel-packed pipettes, elution of absorbed produce with 1:1 EtOAc/Hexanes, and evaporation to give a light yellow oil (445 mg, 81\%).

\section{4-Iodo-3-trimethylsilanylethynyl-benzoic acid 2-[2-(2-methoxy-ethoxy)-ethoxy]-} ethyl ester (10) This compound was prepared by microwave synthesis in 8 Biotage $2-5 \mathrm{~mL}$ vials. Each vial was filled with $250 \mathrm{mg}$ of 8 (0.54 mmol, $1.0 \mathrm{eq}), 6.8 \mathrm{mg}$ of $\mathrm{I}_{2}(27 \mu \mathrm{mol}, 0.05 \mathrm{eq})$, and $10 \mathrm{~g}$ of MeI $(4.4 \mathrm{~mL}, 71 \mathrm{mmol}, \approx 130 \mathrm{eq})$, a stirbar was added, and a septum crimped on. Microwave heat was applied to each tube, at a

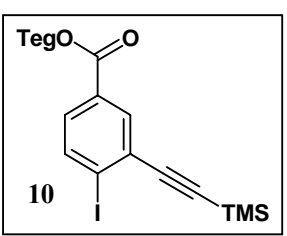
temperature of $150^{\circ} \mathrm{C}$ for a time of one hour. After all reactions had completed, the tubes were opened, combined, filtered through a Celite pad and washed with ethyl ether, and evaporated under a $\mathrm{N}_{2}$ stream. The residue was dissolved in $100 \mathrm{~mL}$ EtOAc, washed with $20 \mathrm{~mL}$ of $5 \%$ aqueous $\mathrm{Na}_{2} \mathrm{SO}_{3}$, dried over $\mathrm{MgSO}_{4}$, and evaporated to yield crude product as a brown oil. Purification by flash chromatography in 20->40\% EtOAc/Hexanes gave a yellow oil $(2.12 \mathrm{~g}, 86 \%) .{ }^{1} \mathrm{H} \mathrm{NMR}\left(\mathrm{CDCl}_{3}\right): \delta$ $8.093(\mathrm{~d}, 1 \mathrm{H}$, phenyl $\mathrm{H}, \mathrm{J}=2.0), 7.926(\mathrm{~d}, 1 \mathrm{H}$, phenyl $\mathrm{H}, \mathrm{J}=8.4), 7.622\left(\mathrm{dd}, 1 \mathrm{H}\right.$, phenyl $\mathrm{J}_{1}=2.0 \mathrm{~J}_{2}=$ 8.4), $4.471\left(\mathrm{~m}, 2 \mathrm{H}, \mathrm{CO}_{2} \mathrm{CH}_{2}\right), 3.83\left(\mathrm{~m}, 2 \mathrm{H}, \mathrm{CH}_{2}\right), 3.67\left(\mathrm{~m}, 6 \mathrm{H}, \mathrm{CH}_{2}\right), 3.52\left(\mathrm{~m}, 2 \mathrm{H}, \mathrm{CH}_{2}\right), 3.367(\mathrm{~s}, 3 \mathrm{H}$, $\left.\mathrm{OCH}_{3}\right), 0.260\left(\mathrm{~s}, 9 \mathrm{H}, \mathrm{Si}\left(\mathrm{CH}_{3}\right)_{3}\right) \mathrm{ppm} .{ }^{13} \mathrm{C} \mathrm{NMR}\left(\mathrm{CDCl}_{3}\right): \delta 165.63,139.12,133.57,130.32,130.15$, $130.12,107.58,105.69,100.20,72.06,70.81,70.78,70.76,69.23,64.60,59.21,-0.13 \mathrm{ppm} \mathrm{MS} \mathrm{m} / \mathrm{z}=$ $490\left(\mathrm{~m}+\mathrm{H}^{+}\right)$.

TMS-Es 2 -Triazene (11) $1.05 \mathrm{~g}$ of $\mathbf{8}(2.26 \mathrm{mmol}, 1.0 \mathrm{eq})$ was converted to 9 with the general TMS deprotection procedure described above. The product was not fully characterized, but was taken directly through Sonogashira coupling using the general procedure described above. The 9, $1.53 \mathrm{~g}$ of 10 (3.69 mmol, $1.6 \mathrm{eq}), 78 \mathrm{mg}$ of $\mathrm{Pd}\left(\mathrm{P}_{3}\right)_{2} \mathrm{Cl}_{2}(111 \mu \mathrm{mol}$,

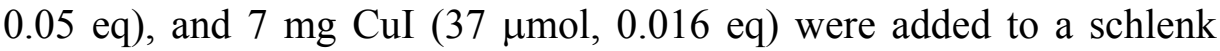

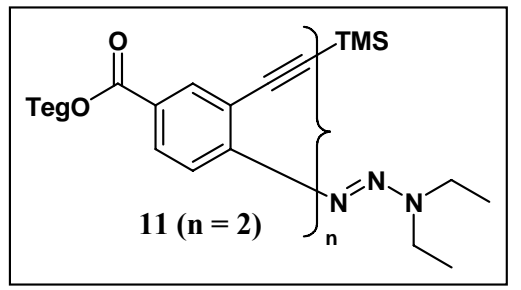
flask with $60 \mathrm{~mL}$ of TEA and $60 \mathrm{~mL}$ of THF. The reaction was heated at $55^{\circ} \mathrm{C}$ overnight, worked up as described in the general procedure, and purified by flash chromatography in $0->10 \%$ acetone $/ \mathrm{CHCl}_{3}$ to obtain a yellow oil $(1.38 \mathrm{~g}, 81 \%) .{ }^{1} \mathrm{H} \mathrm{NMR}\left(\mathrm{CDCl}_{3}\right): \delta 8.249(\mathrm{~d}, 1 \mathrm{H}$, phenyl $\mathrm{H}, \mathrm{J}=1.6), 8.171(\mathrm{~d}, 1 \mathrm{H}$, phenyl $\mathrm{H}, \mathrm{J}=1.6), 7.967\left(\mathrm{dd}, 1 \mathrm{H}\right.$, phenyl $\left.\mathrm{H}, \mathrm{J}_{1}=1.6 \mathrm{~J}_{2}=8.8\right), 7.934\left(\mathrm{dd}, 1 \mathrm{H}\right.$, phenyl $\mathrm{H}, \mathrm{J}_{1}=1.6 \mathrm{~J}_{2}=$ 8.0), $7.516(\mathrm{~d}, 1 \mathrm{H}$, phenyl $\mathrm{H}, \mathrm{J}=8.0), 7.508(\mathrm{~d}, 1 \mathrm{H}$, phenyl $\mathrm{H}, \mathrm{J}=8.8), 4.48\left(\mathrm{~m}, 4 \mathrm{H}, \mathrm{CO}_{2} \mathrm{CH}_{2}\right), 3.83(\mathrm{~m}$, $\left.8 \mathrm{H}, \mathrm{CH}_{2}\right), 3.67\left(\mathrm{~m}, 12 \mathrm{H}, \mathrm{CH}_{2}\right), 3.51\left(\mathrm{~m}, 4 \mathrm{H}, \mathrm{CH}_{2}\right), 3.369\left(\mathrm{~s}, 3 \mathrm{H}, \mathrm{OCH}_{3}\right), 3.351\left(\mathrm{~s}, 3 \mathrm{H}, \mathrm{OCH}_{3}\right), 1.30(\mathrm{~m}$, $\left.6 \mathrm{H}, \mathrm{CH}_{3}\right), 0.263\left(\mathrm{~s}, 9 \mathrm{H}, \mathrm{Si}\left(\mathrm{CH}_{3}\right)_{3}\right)$ ppm. ${ }^{13} \mathrm{C} \mathrm{NMR}\left(\mathrm{CDCl}_{3}\right): \delta 165.98,165.61,155.99,134.99,133.53$, $131.68,131.13,131.04,129.17,129.11,126.16,125.82,117.73,116.69,102.65,99.99,94.76,92.00$, 76.86, 72.06, 70.84, 70.81, 70.79, 70.76, 69.34, 69.30, 64.51, 64.05, 59.19, 59.17, 49.80, 42.56, 14.63, 11.04, $0.05 \mathrm{ppm} \mathrm{MS} \mathrm{m/z}=754\left(\mathrm{~m}+\mathrm{H}^{+}\right)$. 
TMS-Es 2 -Iodide (12) $274 \mathrm{mg}$ of 11 (363 $\mu \mathrm{mol}, 1.0 \mathrm{eq}), 2.4 \mathrm{~mL}$ MeI (5.5g, $0.15 \mathrm{M}$ in 18a) and $2.1 \mathrm{mg} \mathrm{I}_{2}(18 \mu \mathrm{mol}, 0.05 \mathrm{eq})$ were reacted in a schlenk flask under $\mathrm{N}_{2}$ at $110^{\circ} \mathrm{C}$ for 2 weeks. Reaction was filtered through a Celite pad, washed with ethyl ether, and evaporated under a $\mathrm{N}_{2}$ stream. The residue was dissolved in $15 \mathrm{~mL}$ EtOAc, washed with $3 \mathrm{~mL}$ of $5 \%$ aqueous $\mathrm{Na}_{2} \mathrm{SO}_{3}$, dried over $\mathrm{MgSO}_{4}$, and evaporated to yield crude product as a brown oil. Purification by flash chromatography in a 40\%- $>80 \%$ EtOAc/Hexanes gradient gave a dark yellow oil (212 mg, 75\%). ${ }^{1} \mathrm{H}$ NMR (CDCl3): $\delta 8.20(\mathrm{~m}, 2 \mathrm{H}$, phenyl H), $7.97(\mathrm{~m}, 2 \mathrm{H}$, phenyl H), $7.69(\mathrm{~m}, 2 \mathrm{H}$, phenyl $\mathrm{H}), 4.49\left(\mathrm{~m}, 4 \mathrm{H}, \mathrm{CO}_{2} \mathrm{CH}_{2}\right), 3.84\left(\mathrm{~m}, 4 \mathrm{H}, \mathrm{CH}_{2}\right), 3.68\left(\mathrm{~m}, 12 \mathrm{H}, \mathrm{CH}_{2}\right)$, $3.54\left(\mathrm{~m}, 4 \mathrm{H}, \mathrm{CH}_{2}\right), 3.377\left(\mathrm{~s}, 3 \mathrm{H}, \mathrm{OCH}_{3}\right), 3.357\left(\mathrm{~s}, 3 \mathrm{H}, \mathrm{OCH}_{3}\right), 0.283\left(\mathrm{~s}, 9 \mathrm{H}, \mathrm{Si}\left(\mathrm{CH}_{3}\right)_{3}\right) \mathrm{ppm} .{ }^{13} \mathrm{C} \mathrm{NMR}$ $\left(\mathrm{CDCl}_{3}\right): \delta 165.46,139.30,133.77,133.74,132.30,130.59,130.38,130.05,129.60,129.21,125.95$, 107.16, 102.37, 100.47, 97.00, 91.88, 72.07, 72.06, 70.82, 70.80, 70.77, 69.27, 69.18, 64.63, 64.57, $59.21,59.19,0.10 \mathrm{ppm} \mathrm{MS} \mathrm{m} / \mathrm{z}=781\left(\mathrm{~m}+\mathrm{H}^{+}\right)$.

H-Es 2 -Triazene (13) The general TMS deprotection procedure, listed above, was used to prepare this compound. $422 \mathrm{mg}$ of $11(560 \mu \mathrm{mol}$, $1.0 \mathrm{eq})$ was dissolved in $4.0 \mathrm{~mL} \mathrm{THF}$, cooled to $0^{\circ} \mathrm{C}$, and reacted with $0.671 \mathrm{~mL}(1.6 \mathrm{eq})$ of $1 \mathrm{M} \mathrm{TBAF} / \mathrm{THF}+5 \% \mathrm{H}_{2} \mathrm{O}$. After 5 minutes, 4.0 $\mathrm{mL}$ of hexane was added to the reaction. After another 10 minutes of stirring, reaction mixture was injected onto a silica-packed pipette and eluted with $150 \mathrm{~mL} \mathrm{2:1} \mathrm{EtOAc/Hexanes.} \mathrm{Evaporation} \mathrm{gave} \mathrm{a} \mathrm{yellow-}$

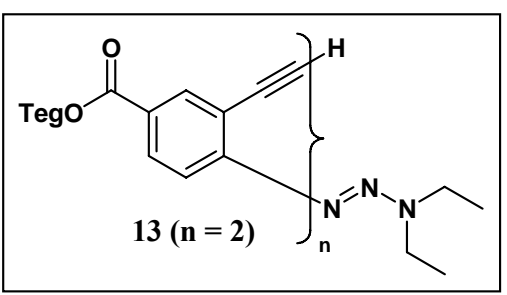
orange oil (317 mg, 83\%).

TMS-Es 3 -Triazene (14) This compound was prepared by the general Sonogashira procedure described above. $317 \mathrm{mg}$ of 13 (465 $\mu \mathrm{mol}, 1.2$ eq), $190 \mathrm{mg}$ of 10 (387 $\mu \mathrm{mol}, 1.0 \mathrm{eq}), 8.1 \mathrm{mg}$ of $\mathrm{Pd}\left(\mathrm{P}_{\phi_{3}}\right)_{2} \mathrm{Cl}_{2}(12 \mu \mathrm{mol}$,

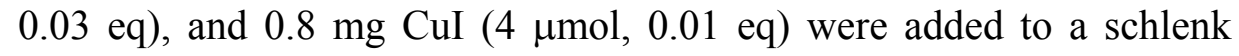
flask in $15 \mathrm{~mL}$ TEA and $30 \mathrm{~mL}$ THF. The reaction was heated at $55^{\circ} \mathrm{C}$ overnight, worked up as described in the general procedure, and purified by flash chromatography in $0->20 \%$ acetone $/ \mathrm{CHCl}_{3}$ to obtain an orange

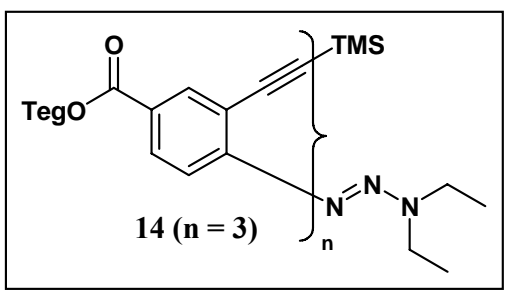
oil (350 mg, 87\%). ${ }^{1} \mathrm{H}$ NMR $\left(\mathrm{CDCl}_{3}\right): \delta 8.284(\mathrm{~d}, 1 \mathrm{H}$, phenyl $\mathrm{H}, \mathrm{J}=2.0), 8.265(\mathrm{~d}, 1 \mathrm{H}$, phenyl $\mathrm{H}, \mathrm{J}=$ 2.0), $8.169(\mathrm{~d}, 1 \mathrm{H}$, phenyl $\mathrm{H}, \mathrm{J}=2.0), 8.01\left(\mathrm{dd}, 1 \mathrm{H}\right.$, phenyl $\left.\mathrm{H}, \mathrm{J}_{1}=2.0 \mathrm{~J}_{2}=8.4\right), 7.98(\mathrm{dd}, 1 \mathrm{H}$, phenyl $\mathrm{H}$, $\left.\mathrm{J}_{1}=2.0 \mathrm{~J}_{2}=8.4\right), 7.90\left(\mathrm{dd}, 1 \mathrm{H}\right.$, phenyl $\left.\mathrm{H}, \mathrm{J}_{1}=2.0 \mathrm{~J}_{2}=8.4\right), 7.607(\mathrm{~d}, 1 \mathrm{H}$, phenyl $\mathrm{H}, \mathrm{J}=8.4), 7.575(\mathrm{~d}$, $1 \mathrm{H}$, phenyl $\mathrm{H}, \mathrm{J}=8.4), 7.511(\mathrm{~d}, 1 \mathrm{H}$, phenyl $\mathrm{H}, \mathrm{J}=8.4), 4.49\left(\mathrm{~m}, 6 \mathrm{H}, \mathrm{CO}_{2} \mathrm{CH}_{2}\right), 3.6-3.9\left(\mathrm{~m}, 30 \mathrm{H}, \mathrm{CH}_{2}\right)$, $3.53\left(\mathrm{~m}, 4 \mathrm{H}, \mathrm{CH}_{2}\right), 3.360\left(\mathrm{~s}, 1 \mathrm{H}, \mathrm{OCH}_{3}\right), 3.341\left(\mathrm{~s}, 1 \mathrm{H}, \mathrm{OCH}_{3}\right), 1.2-1.3\left(\mathrm{~m}, 6 \mathrm{H}, \mathrm{CH}_{3}\right), 0.285(\mathrm{~s}, 9 \mathrm{H}$, $\left.\mathrm{Si}\left(\mathrm{CH}_{3}\right)_{3}\right) \mathrm{ppm} .{ }^{13} \mathrm{C} \mathrm{NMR}\left(\mathrm{CDCl}_{3}\right): \delta$ values $165.82,165.37,165.31,155.98,135.03,133.29,132.37$, 131.79 , 131.00, 130.93, 130.07, 129.57, 129.49, 129.33, 129.16, 126.08, 125.80, 125.36, 117.40, $116.80,102.29,100.16,95.21,94.35,92.24,91.80,71.94,70.68,70.64,70.60,69.22,69.14,69.12$, $64.42,64.33,64.04,59.06,59.03,49.54,14.42,10.91,0.11 \mathrm{ppm} \mathrm{MS} \mathrm{m} / \mathrm{z}=1044\left(\mathrm{~m}+\mathrm{H}^{+}\right)$. 
H-Es 3 -Triazene (14a) This compound was prepared by the general TMS deprotection procedure described above. $350 \mathrm{mg}$ of $\mathbf{1 4}(335 \mu \mathrm{mol}$, $1.0 \mathrm{eq})$ was dissolved in $14 \mathrm{~mL}$ THF, cooled to $0^{\circ} \mathrm{C}$, and reacted with $0.402 \mathrm{~mL}(1.2 \mathrm{eq})$ of $1 \mathrm{M} \mathrm{TBAF} / \mathrm{THF}+5 \% \mathrm{H}_{2} \mathrm{O}$. After 5 minutes, 14 $\mathrm{mL}$ hexane was added and the reaction was stirred for another 10 minutes. The reaction mixture was filtered through silica gel-packed pipettes and eluted with 2:1 EtOAc/Hexanes. Evaporation gave a

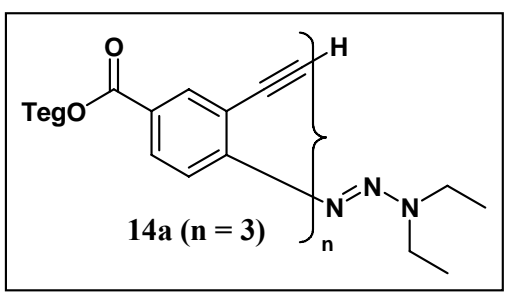
yellow oil (305 mg, 94\%).

TMS-Es 4 -Triazene (1) This compound was prepared by the general Sonogashira procedure described above. $256 \mathrm{mg}$ of $14 \mathrm{a}(263 \mu \mathrm{mol}, 1.0$ eq), $194 \mathrm{mg}$ of 10 (395 $\mu \mathrm{mol}, 1.5 \mathrm{eq}), 5.5 \mathrm{mg}$ of $\mathrm{Pd}\left(\mathrm{P}_{3}\right)_{2} \mathrm{Cl}_{2}(8 \mu \mathrm{mol}$, $0.03 \mathrm{eq})$, and $0.5 \mathrm{mg}$ of $\mathrm{CuI}(3 \mu \mathrm{mol}, 0.01 \mathrm{eq})$ were added to a schlenk flask in $15 \mathrm{~mL}$ TEA and $30 \mathrm{~mL}$ THF. The reaction was heated at $55^{\circ} \mathrm{C}$ overnight, worked up as described in the general procedure, and purified by flash chromatography in $0->40 \%$ acetone $/ \mathrm{CHCl}_{3}$ to obtain a

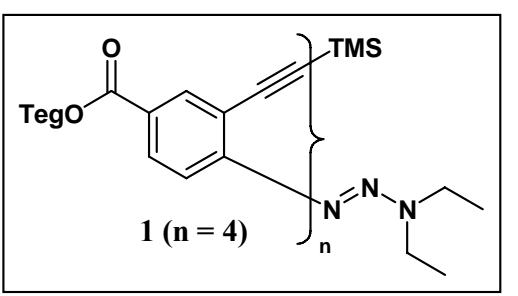
light yellow oil $(284 \mathrm{mg}, 77 \%)$. ${ }^{1} \mathrm{H} \mathrm{NMR}\left(\mathrm{CDCl}_{3}\right): \delta 8.299(\mathrm{~d}, 1 \mathrm{H}$, phenyl $\mathrm{H}, \mathrm{J}=2.0), 8.253(\mathrm{~d}, 1 \mathrm{H}$, phenyl $\mathrm{H}, \mathrm{J}=2.0), 8.196(\mathrm{~d}, 1 \mathrm{H}$, phenyl $\mathrm{H}, \mathrm{J}=2.0), 8.110(\mathrm{~d}, 1 \mathrm{H}$, phenyl $\mathrm{H}, \mathrm{J}=2.0), 8.011(\mathrm{dd}, 1 \mathrm{H}$, phenyl $\left.\mathrm{H}, \mathrm{J}_{1}=2.0 \mathrm{~J}_{2}=8.4\right), 7.983\left(\mathrm{dd}, 1 \mathrm{H}\right.$, phenyl $\left.\mathrm{H}, \mathrm{J}_{1}=2.0 \mathrm{~J}_{2}=8.4\right), 7.926\left(\mathrm{dd}, 1 \mathrm{H}\right.$, phenyl $\mathrm{H}, \mathrm{J}_{1}=$ $\left.2.0 \mathrm{~J}_{2}=8.4\right), 7.866\left(\mathrm{dd}, 1 \mathrm{H}\right.$, phenyl $\left.\mathrm{H}, \mathrm{J}_{1}=2.0 \mathrm{~J}_{2}=8.4\right), 7.668(\mathrm{~d}, 1 \mathrm{H}$, phenyl $\mathrm{H}, \mathrm{J}=8.4), 7.615(\mathrm{~d}, 1 \mathrm{H}$, phenyl $\mathrm{H}, \mathrm{J}=8.4), 7.593(\mathrm{~d}, 1 \mathrm{H}$, phenyl $\mathrm{H}, \mathrm{J}=8.4), 7.451(\mathrm{~d}, 1 \mathrm{H}$, phenyl $\mathrm{H}, \mathrm{J}=8.4), 4.43-4.56(\mathrm{~m}, 8 \mathrm{H}$, $\left.\mathrm{CO}_{2} \mathrm{CH}_{2}\right), 3.6-3.9\left(\mathrm{~m}, 36 \mathrm{H}, \mathrm{CH}_{2}\right), 3.48-3.58\left(\mathrm{~m}, 8 \mathrm{H}, \mathrm{CH}_{2}\right), 3.32-3.38\left(\mathrm{~m}, 12 \mathrm{H}, \mathrm{OCH}_{3}\right), 1.18-1.27(\mathrm{~m}$, $\left.6 \mathrm{H}, \mathrm{CH}_{3}\right), 0.280\left(\mathrm{~s}, 9 \mathrm{H}, \mathrm{Si}\left(\mathrm{CH}_{3}\right)_{3}\right) \mathrm{ppm} .{ }^{13} \mathrm{C} \mathrm{NMR}(\mathrm{CDCl} 3): \delta 165.73,165.30,165.25,165.20,155.82$, $135.02,133.61,133.25,132.51,132.17,131.95,130.94,130.81,129.90,129.81,129.74,129.55$, $129.36,129.10,125.93,125.82,125.53,125.17,117.32,116.68,102.29,100.00,95.37,94.87,93.75$, $92.51,92.14,91.70,71.92,70.68,70.64,70.60,69.22,69.15,69.11,69.08,64.38,64.01,59.05,59.03$, 49.50, 42.27, 14.42, 10.93, $-0.11 \mathrm{ppm}$. MS $\mathrm{m} / \mathrm{z}=1335\left(\mathrm{~m}+\mathrm{H}^{+}\right)$.

TMS-Es5-Triazene (2) This compound was prepared by the general Sonogashira procedure described above. $234 \mathrm{mg}$ of 14a $(241 \mu \mathrm{mol}, 1.0$ eq) , $207 \mathrm{mg}$ of 12 (265 $\mu \mathrm{mol}, 1.1 \mathrm{eq}), 8.5 \mathrm{mg}$ of $\mathrm{Pd}\left(\mathrm{P}_{3}\right)_{2} \mathrm{Cl}_{2}(12 \mu \mathrm{mol}$, $0.05 \mathrm{eq})$, and $0.5 \mathrm{mg}$ of $\mathrm{CuI}(2.4 \mu \mathrm{mol}, 0.01 \mathrm{eq})$ were added to a schlenk flask in $50 \mathrm{~mL}$ TEA and $100 \mathrm{~mL}$ THF. The reaction was heated at $55^{\circ} \mathrm{C}$ overnight, worked up as described in the general procedure, and purified

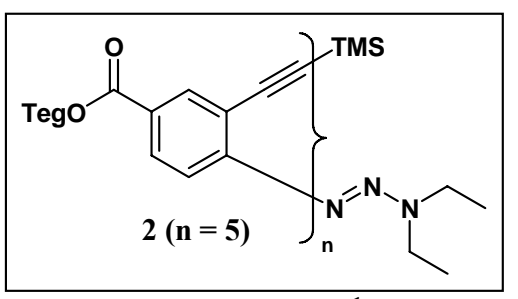
by flash chromatography in $0->30 \%$ acetone $/ \mathrm{CHCl}_{3}$ to obtain an orange oil $(269 \mathrm{mg}, 69 \%)$. ${ }^{1} \mathrm{H}$ NMR $\left(\mathrm{CD}_{3} \mathrm{CN}\right): \delta 8.203(\mathrm{~d}, 1 \mathrm{H}$, phenyl $\mathrm{H}, \mathrm{J}=2.0), 8.019\left(\mathrm{dd}, 1 \mathrm{H}\right.$, phenyl $\left.\mathrm{H}, \mathrm{J}_{1}=2.0 \mathrm{~J}_{2}=8.4\right), 7.948(\mathrm{~d}, 1 \mathrm{H}$, phenyl $\mathrm{H}, \mathrm{J}=2.0), 7.843\left(\mathrm{dd}, 1 \mathrm{H}\right.$, phenyl $\left.\mathrm{H}, \mathrm{J}_{1}=2.0 \mathrm{~J}_{2}=8.4\right), 7.835(\mathrm{~d}, 1 \mathrm{H}$, phenyl $\mathrm{H}, \mathrm{J}=2.0), 7.832$ $(\mathrm{d}, 1 \mathrm{H}$, phenyl $\mathrm{H}, \mathrm{J}=2.0), 7.779(\mathrm{~d}, 1 \mathrm{H}$, phenyl $\mathrm{H}, \mathrm{J}=8.4), 7.771(\mathrm{~d}, 1 \mathrm{H}$, phenyl $\mathrm{H}, \mathrm{J}=2.0), 7.766(\mathrm{dd}$, $1 \mathrm{H}$, phenyl $\left.\mathrm{H}, \mathrm{J}_{1}=2.0 \mathrm{~J}_{2}=8.4\right), 7.753\left(\mathrm{dd}, 1 \mathrm{H}\right.$, phenyl $\left.\mathrm{H}, \mathrm{J}_{1}=2.0 \mathrm{~J}_{2}=8.4\right), 7.645\left(\mathrm{dd}, 1 \mathrm{H}\right.$, phenyl $\mathrm{H}, \mathrm{J}_{1}$ $\left.=2.0 \mathrm{~J}_{2}=8.4\right), 7.510(\mathrm{~d}, 1 \mathrm{H}$, phenyl $\mathrm{H}, \mathrm{J}=8.4), 7.426(\mathrm{~d}, 1 \mathrm{H}$, phenyl $\mathrm{H}, \mathrm{J}=8.4), 7.318(\mathrm{~d}, 1 \mathrm{H}$, phenyl $\mathrm{H}$, $\mathrm{J}=8.4), 7.304(\mathrm{~d}, 1 \mathrm{H}$, phenyl $\mathrm{H}, \mathrm{J}=8.4), 4.35-4.48\left(\mathrm{~m}, 10 \mathrm{H}, \mathrm{CO}_{2} \mathrm{CH}_{2}\right), 3.35-3.80\left(\mathrm{~m}, 54 \mathrm{H}, \mathrm{CH}_{2}\right), 3.20-$ $3.28\left(\mathrm{~m}, 15 \mathrm{H}, \mathrm{OCH}_{3}\right), 1.10-1.30\left(\mathrm{~m}, 6 \mathrm{H}, \mathrm{CH}_{3}\right), 0.235\left(\mathrm{~s}, 9 \mathrm{H}, \mathrm{Si}\left(\mathrm{CH}_{3}\right)_{3}\right) \mathrm{ppm} .{ }^{13} \mathrm{C} \mathrm{NMR}\left(\mathrm{CD}_{3} \mathrm{CN}\right): \delta$ $166.56,166.26,166.16,166.08,166.05,157.03,136.21,134.41,134.14,133.95,133.82,133.76$, $133.42,131.89,131.84,131.70,131.19,131.18,130.93,130.92,130.75,130.64,130.53,130.52$, $130.42,130.16,127.01,126.87,126.70,126.45,126.04,118.46,117.63,103.47,101.25,96.39,96.06$, $95.29,95.21,93.81,93.17,93.15,93.00,73.00,72.96,71.73,71.72,71.69,71.66,71.48,71.46,71.44$, $71.40,71.39,70.02,69.99,69.97,65.99,65.91,65.86,65.82,65.29,59.31,59.27,50.78,47.98,43.54$, 15.06, 11.72, 0.45 ppm. 
H-Es 4 -Triazene (1a) This compound was prepared by the general TMS deprotection procedure described above. $146 \mathrm{mg}$ of 1 (109 $\mu \mathrm{mol}, 1.0$ eq) was dissolved in $6 \mathrm{~mL} \mathrm{THF}$, cooled to $0^{\circ} \mathrm{C}$, and reacted with 0.120 $\mathrm{mL}(120 \mu \mathrm{mol}, 1.1 \mathrm{eq})$ of $1 \mathrm{M} \mathrm{TBAF} / \mathrm{THF}+5 \% \mathrm{H}_{2} \mathrm{O}$. After 5 minutes, $6 \mathrm{~mL}$ hexane was added and the reaction was stirred for another 10 minutes. The reaction mixture was injected onto a dry $12 \mathrm{~g}$ ISCO silica cartridge and eluted with a $0->20 \%$ acetone $/ \mathrm{CHCl}_{3}$ gradient to obtain a

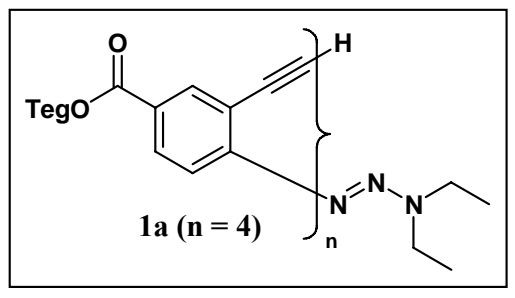
light yellow oil (101 mg, 73\%).

TMS-Es 6 -Triazene (3) This compound was prepared by the general Sonogashira procedure described above. $101 \mathrm{mg}$ of $\mathbf{1 a}(75.7 \mu \mathrm{mol}, 1.0$ eq), $64.9 \mathrm{mg}$ of $12(83.2 \mu \mathrm{mol}, 1.1 \mathrm{eq}), 1.6 \mathrm{mg}$ of $\mathrm{Pd}\left(\mathrm{P}_{3}\right)_{2} \mathrm{Cl}_{2}(2.3$ $\mu \mathrm{mol}, 0.03 \mathrm{eq})$, and $0.2 \mathrm{mg}$ of $\mathrm{CuI}(0.7 \mu \mathrm{mol}, 0.01 \mathrm{eq})$ were added to a schlenk flask in $5.5 \mathrm{~mL}$ TEA and $11 \mathrm{~mL}$ THF. The reaction was heated at $55^{\circ} \mathrm{C}$ overnight, worked up as described in the general procedure, and purified by flash chromatography in $0->35 \%$ acetone $/ \mathrm{CHCl}_{3}$ to obtain

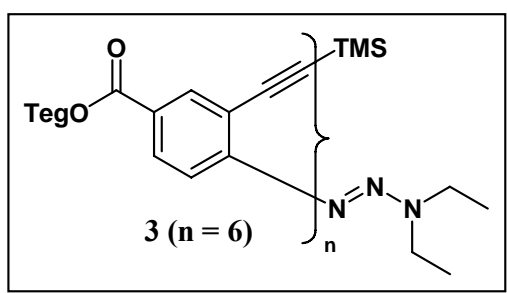
an orange oil $(117 \mathrm{mg}, 81 \%)$. ${ }^{1} \mathrm{H}$ NMR $\left(\mathrm{CD}_{3} \mathrm{CN}\right): \delta 7.934(\mathrm{~d}, 1 \mathrm{H}$, phenyl $\mathrm{H}, \mathrm{J}=2.0), 7.926(\mathrm{~d}, 1 \mathrm{H}$, phenyl $\mathrm{H}, \mathrm{J}=2.0$ ), $7.827\left(\mathrm{dd}, 1 \mathrm{H}\right.$, phenyl $\mathrm{H}, \mathrm{J}_{1}=2.0 \mathrm{~J}_{2}=8.4$ ), $7.759\left(\mathrm{dd}, 1 \mathrm{H}\right.$, phenyl $\mathrm{H}, \mathrm{J}_{1}=2.0 \mathrm{~J}_{2}=$ 8.4), $7.752(\mathrm{~d}, 1 \mathrm{H}$, phenyl $\mathrm{H}, \mathrm{J}=2.0), 7.717(\mathrm{~d}, 1 \mathrm{H}$, phenyl $\mathrm{H}, \mathrm{J}=2.0), 7.713\left(\mathrm{dd}, 1 \mathrm{H}\right.$, phenyl $\mathrm{H}, \mathrm{J}_{1}=$ $\left.2.0 \mathrm{~J}_{2}=8.4\right), 7.703\left(\mathrm{dd}, 1 \mathrm{H}\right.$, phenyl H, $\left.\mathrm{J}_{1}=2.0 \mathrm{~J}_{2}=8.4\right), 7.626(\mathrm{~d}, 1 \mathrm{H}$, phenyl $\mathrm{H}, \mathrm{J}=8.4), 7.598(\mathrm{~d}, 1 \mathrm{H}$, phenyl $\mathrm{H}, \mathrm{J}=2.0), 7.564(\mathrm{~d}, 1 \mathrm{H}$, phenyl $\mathrm{H}, \mathrm{J}=2.0), 7.551\left(\mathrm{dd}, 1 \mathrm{H}\right.$, phenyl $\left.\mathrm{H}, \mathrm{J}_{1}=2.0 \mathrm{~J}_{2}=8.4\right), 7.540$ (dd, $1 \mathrm{H}$, phenyl $\left.\mathrm{H}, \mathrm{J}_{1}=2.0 \mathrm{~J}_{2}=8.4\right), 7.430(\mathrm{~d}, 1 \mathrm{H}$, phenyl $\mathrm{H}, \mathrm{J}=8.4), 7.346(\mathrm{~d}, 1 \mathrm{H}$, phenyl $\mathrm{H}, \mathrm{J}=8.4)$, $7.245(\mathrm{~d}, 1 \mathrm{H}$, phenyl $\mathrm{H}, \mathrm{J}=8.4), 7.162(\mathrm{~d}, 1 \mathrm{H}$, phenyl $\mathrm{H}, \mathrm{J}=8.4), 7.117(\mathrm{~d}, 1 \mathrm{H}$, phenyl $\mathrm{H}, \mathrm{J}=8.4)$, 4.25-4.45 (m, $\left.12 \mathrm{H}, \mathrm{CO}_{2} \mathrm{CH}_{2}\right), 3.35-3.80\left(\mathrm{~m}, 64 \mathrm{H}, \mathrm{CH}_{2}\right), 3.20-3.30\left(\mathrm{~m}, 18 \mathrm{H}, \mathrm{OCH}_{3}\right), 1.15-1.25(\mathrm{~m}, 6 \mathrm{H}$, $\left.\mathrm{CH}_{3}\right), 0.283\left(\mathrm{~s}, 9 \mathrm{H}, \mathrm{Si}\left(\mathrm{CH}_{3}\right)_{3}\right)$ ppm. ${ }^{13} \mathrm{C} \operatorname{NMR}\left(\mathrm{CDCl}_{3}\right): \delta 166.65,166.16,166.09,166.03,165.95$, $165.91,156.83,136.04,133.98,133.86,133.63,133.61,133.53,133.50,133.45,133.29,131.68$, $131.65,130.95,130.82,130.79,130.75,130.74,130.66,130.59,130.35,130.32,130.28,130.23$, $130.18,130.03,126.83,126.62,126.60,126.48,126.25,126.06,118.49,117.40,103.57,101.40,96.15$, $95.79,95.52,95.42,95.18,93.47,93.35,93.31,93.10,92.98,72.97,72.96,72.91,71.72,71.70,71.69$, 71.66, 71.60, 71.47, 71.42, 71.40, 71.38, 71.35, 70.09, 70.08, 70.05, 70.02, 65.93, 65.92, 65.88, 65.77, $65.75,65.29,59.39,59.37,59.36,59.35,59.34,59.32,50.81,47.75,43.57,15.05,11.71,0.57 \mathrm{ppm}$. 


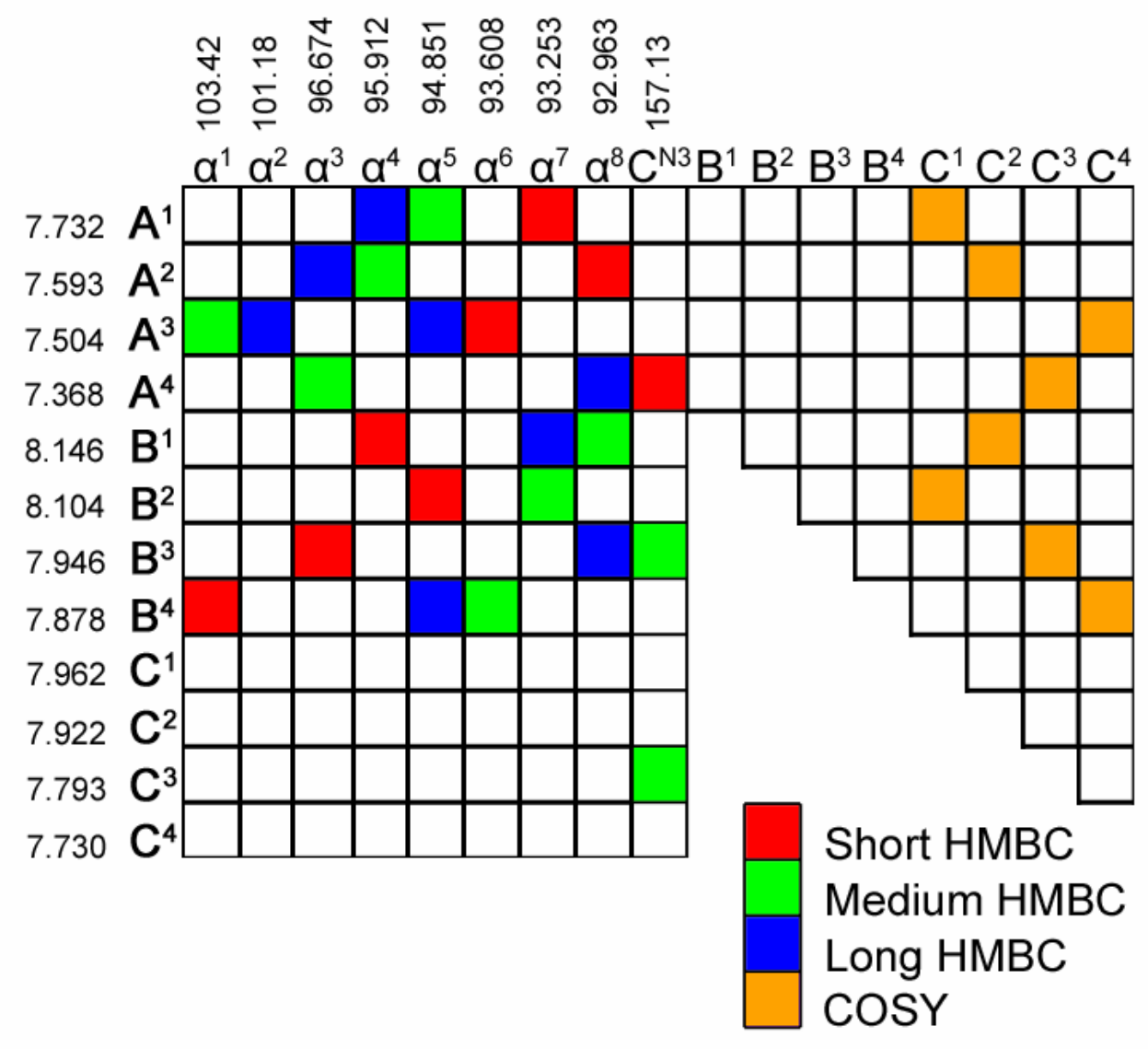

Figure S3. Table of observed HMBC and COSY interactions in Es4. HMBC interactions between A or $\mathrm{B}$ ring protons and adjacent acetylene carbons are classified as short. HMBC interactions longer than 2 bonds, which do not cross a triple bond, are classified as medium. HMBC interactions across a triple bond are classified as long. ${ }^{1} \mathrm{H}$ and ${ }^{13} \mathrm{C}$ chemical shifts of aromatic protons and acetylenic carbons are shown. $\mathrm{C}^{\mathrm{N} 3}$ refers to aromatic carbon with $\mathrm{N}_{3} \mathrm{Et}_{2}$ substituent. 


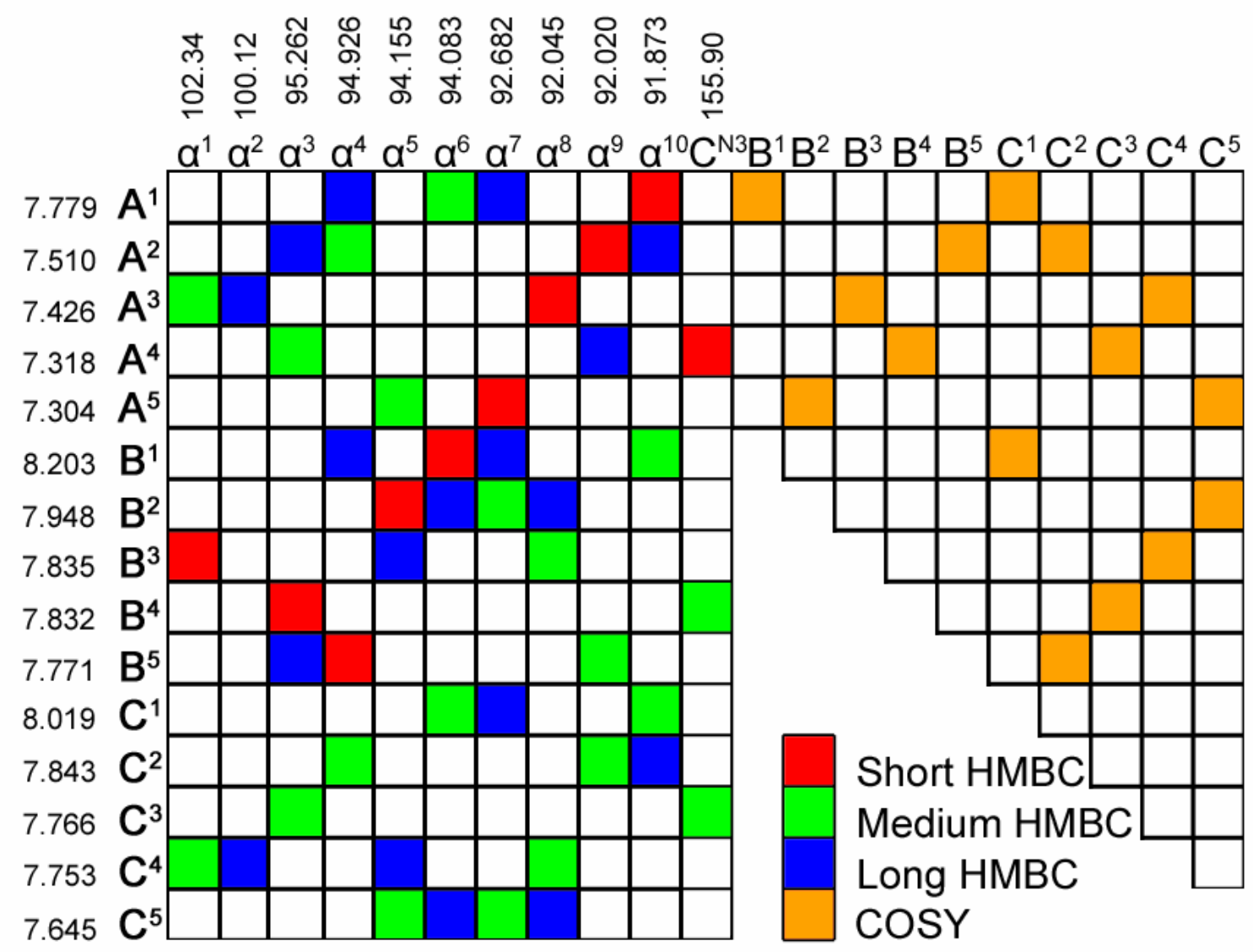

Figure S4. Table of observed HMBC and COSY interactions in Es 5 . HMBC interactions between A or $\mathrm{B}$ ring protons and adjacent acetylene carbons are classified as short. HMBC interactions longer than 2 bonds, which do not cross a triple bond, are classified as medium. HMBC interactions across a triple bond are classified as long. ${ }^{1} \mathrm{H}$ and ${ }^{13} \mathrm{C}$ chemical shifts of aromatic protons and acetylenic carbons are shown. $\mathrm{C}^{\mathrm{N} 3}$ refers to aromatic carbon with $\mathrm{N}_{3} \mathrm{Et}_{2}$ substituent. 

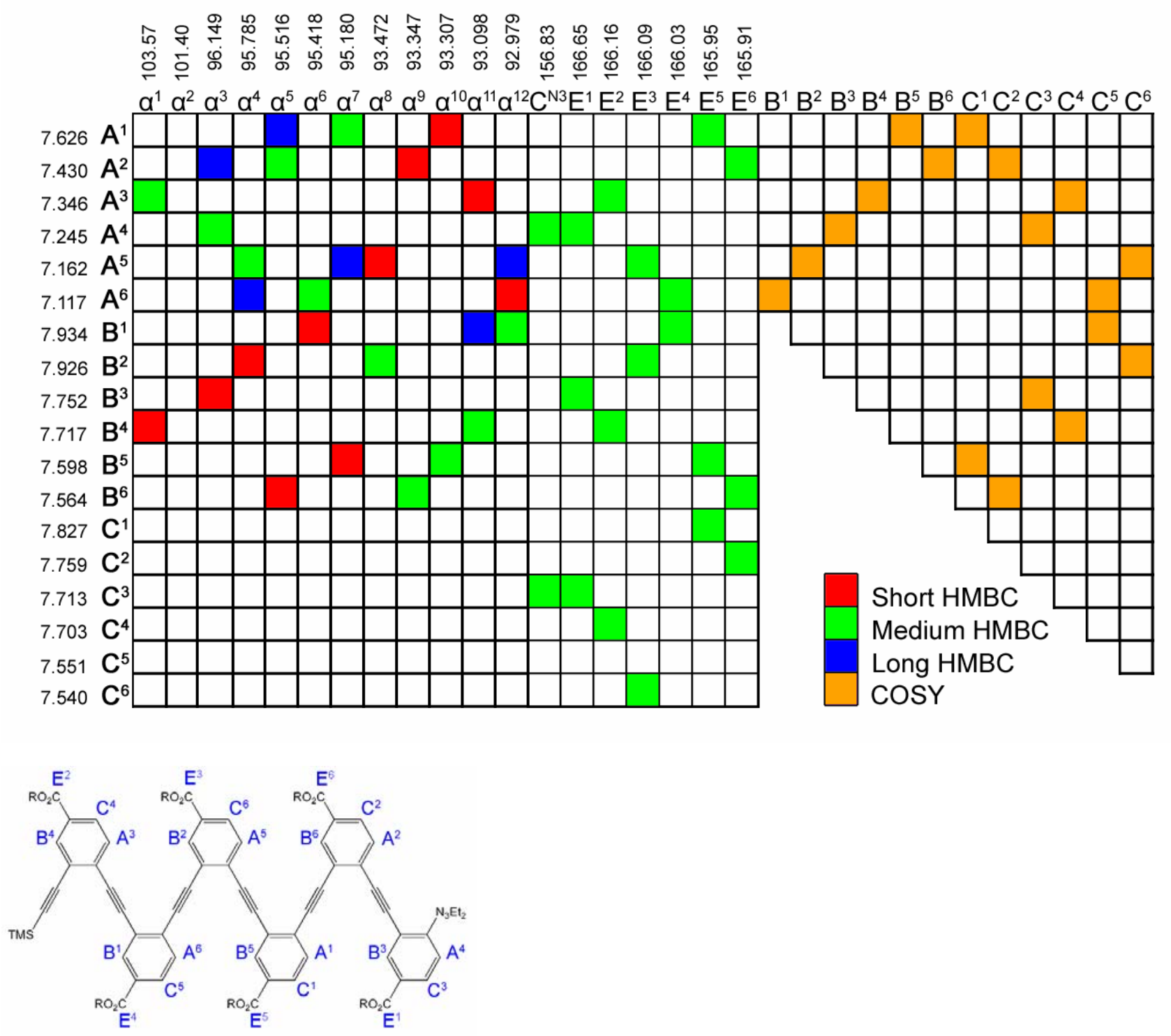

Figure S5. Top panel shows table of observed HMBC and COSY interactions in Es 6 . HMBC interactions between A or B ring protons and adjacent acetylene carbons are classified as short. HMBC interactions longer than 2 bonds, which do not cross a triple bond, are classified as medium. HMBC interactions across a triple bond are classified as long. ${ }^{1} \mathrm{H}$ and ${ }^{13} \mathrm{C}$ chemical shifts of aromatic protons and acetylenic carbons are shown. $\mathrm{C}^{\mathrm{N} 3}$ refers to aromatic carbon with $\mathrm{N}_{3} \mathrm{Et}_{2}$ substituent. $\mathrm{E}^{1-6}$ refers to ester carbons, in order of chemical shift. Bottom panel shows ester carbon assignments. 

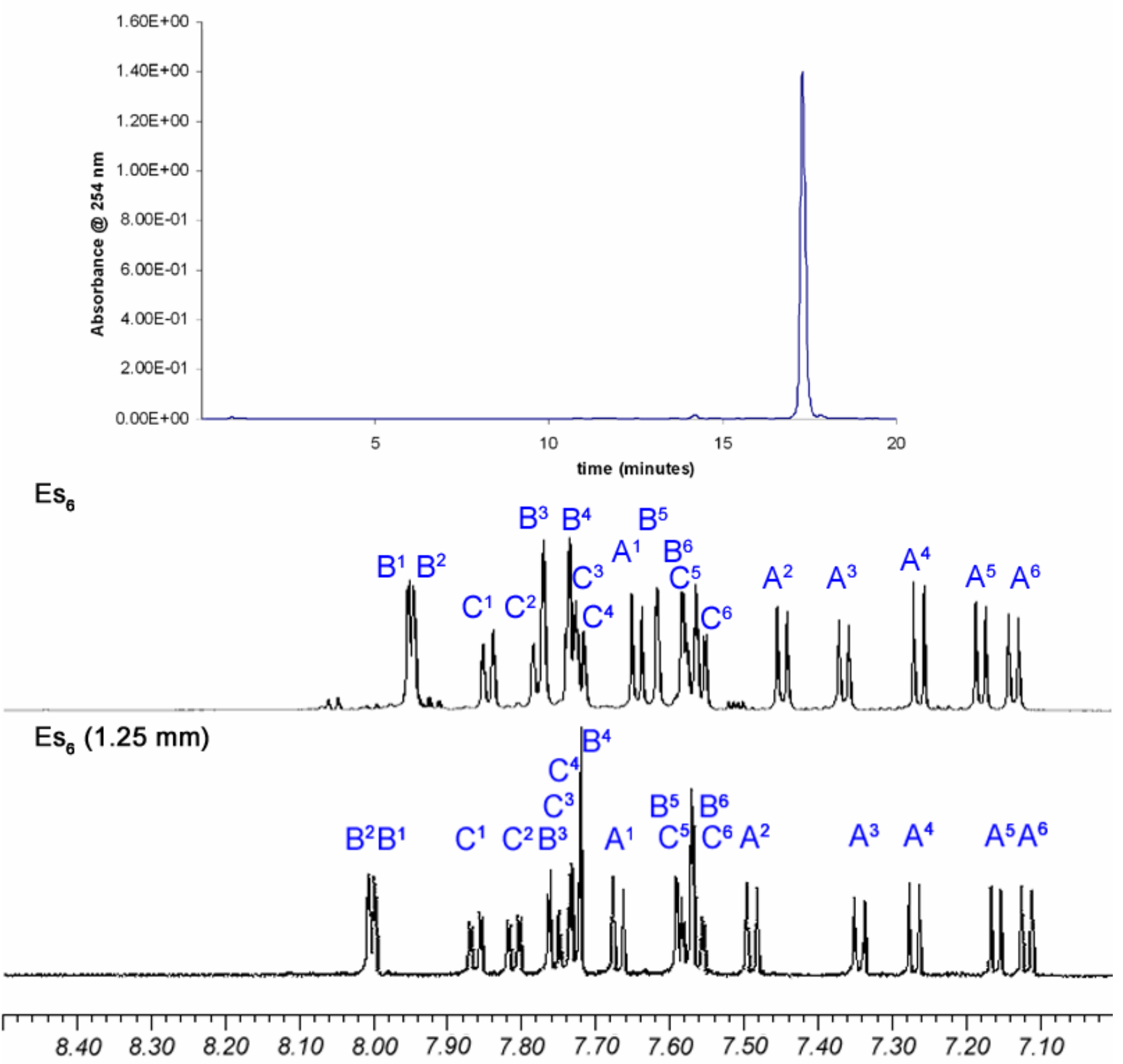

Figure S6. HPLC and NMR measurements confirming purity of $\mathrm{Es}_{6}$. Top panel shows chromatogram of $\mathrm{Es}_{6}$, showing $>95 \%$ purity $(\approx 96 \%)$ by integrated absorbance @ $254 \mathrm{~nm}$. Lower panel shows aromatic region of ${ }^{1} \mathrm{H}$ NMR for $\mathrm{Es}_{6}$ - saturated concentration as used in assignment experiments shown above, while lower $1.25 \mathrm{mM}$ concentration sample shown below. Disappearance of smaller NMR peaks upon dilution provides evidence that these are the result of aggregation at high concentrations, and not of an impurity in the sample. 


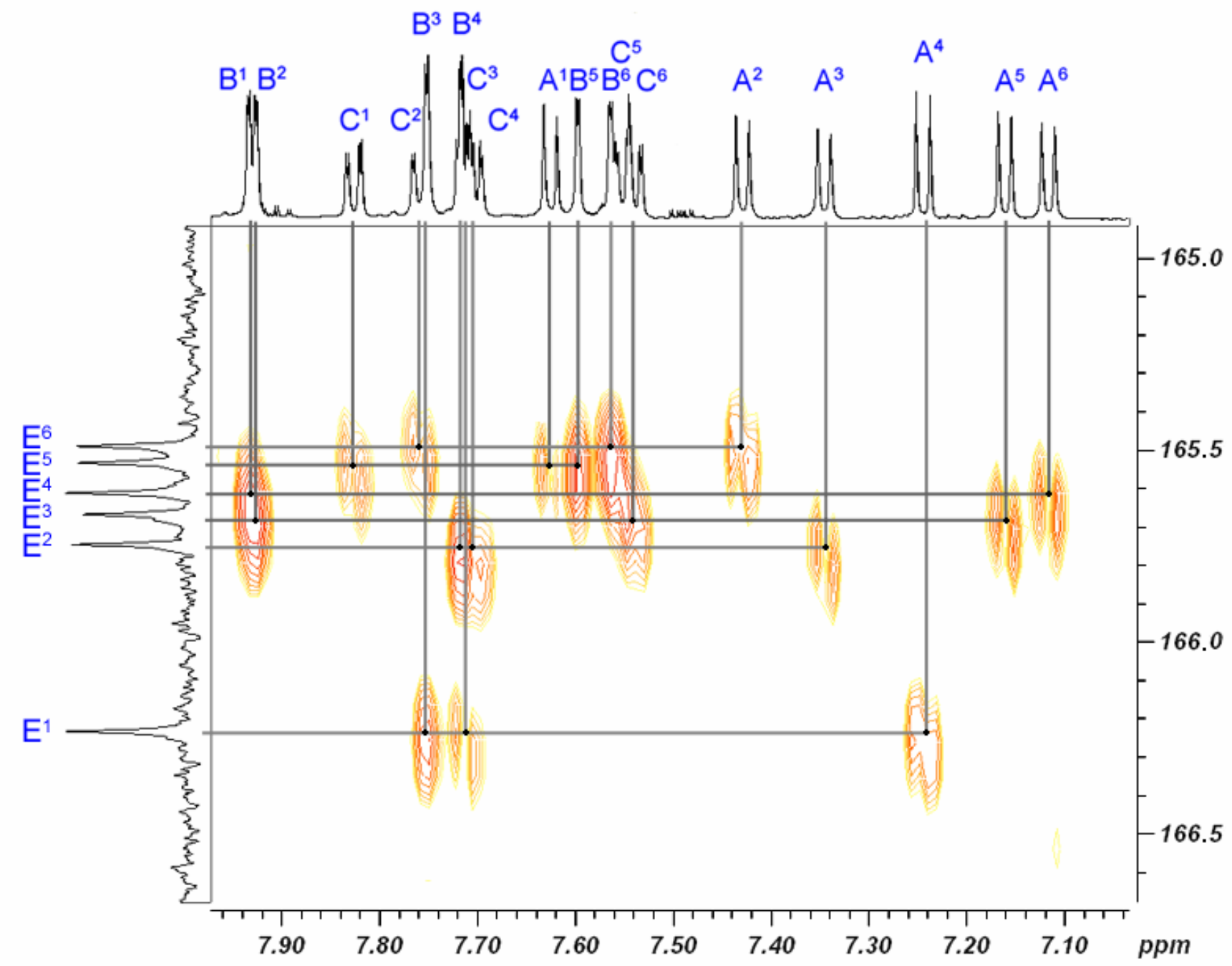

Figure S7. HMBC interactions between A, B, and C protons of Es6 and ester carbons adjacent to each ring. Same $\mathrm{HMBC}$ data set as previously mentioned. 


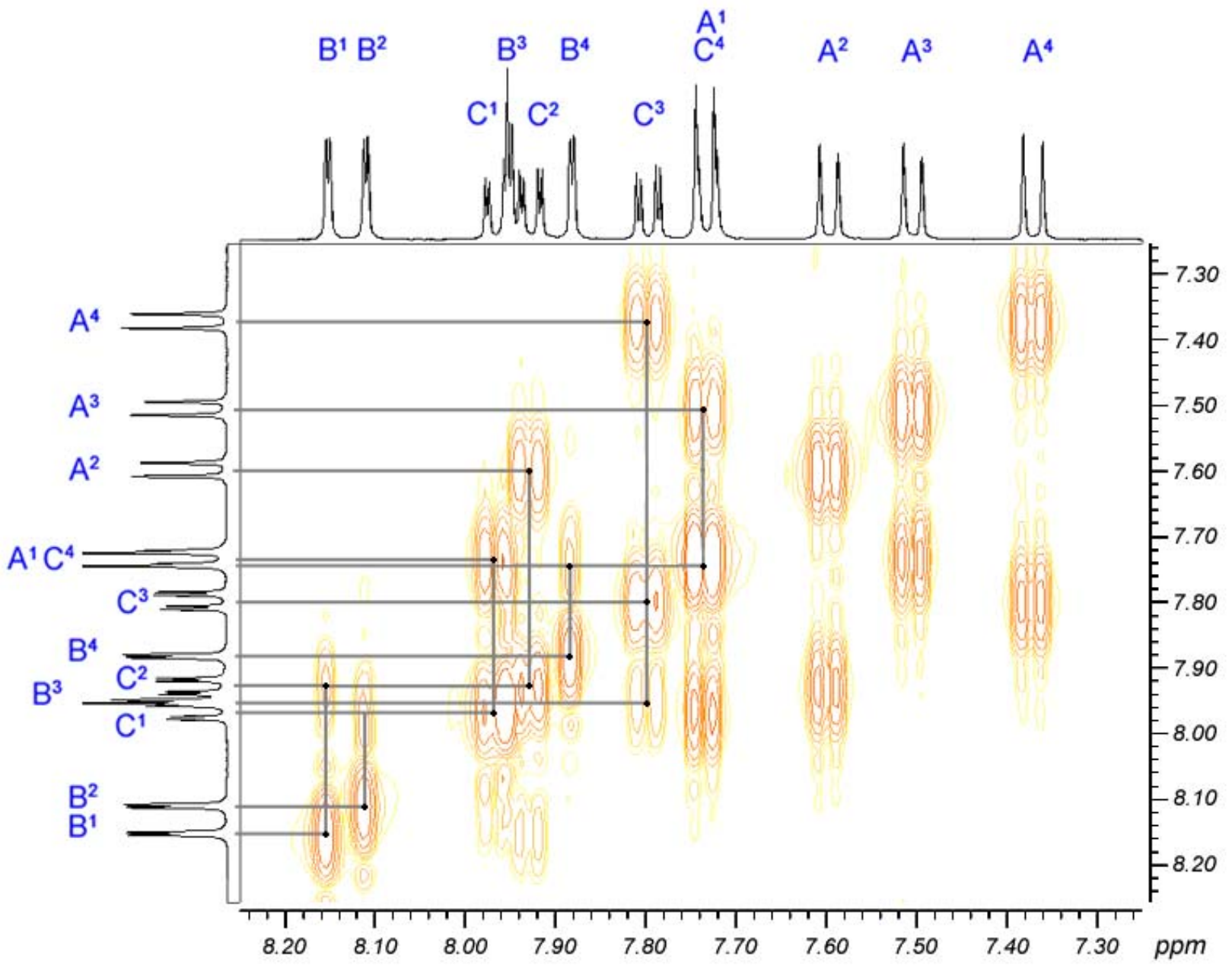

Figure S8. COSY spectrum of $\mathrm{Es}_{4}$, aromatic proton region. 


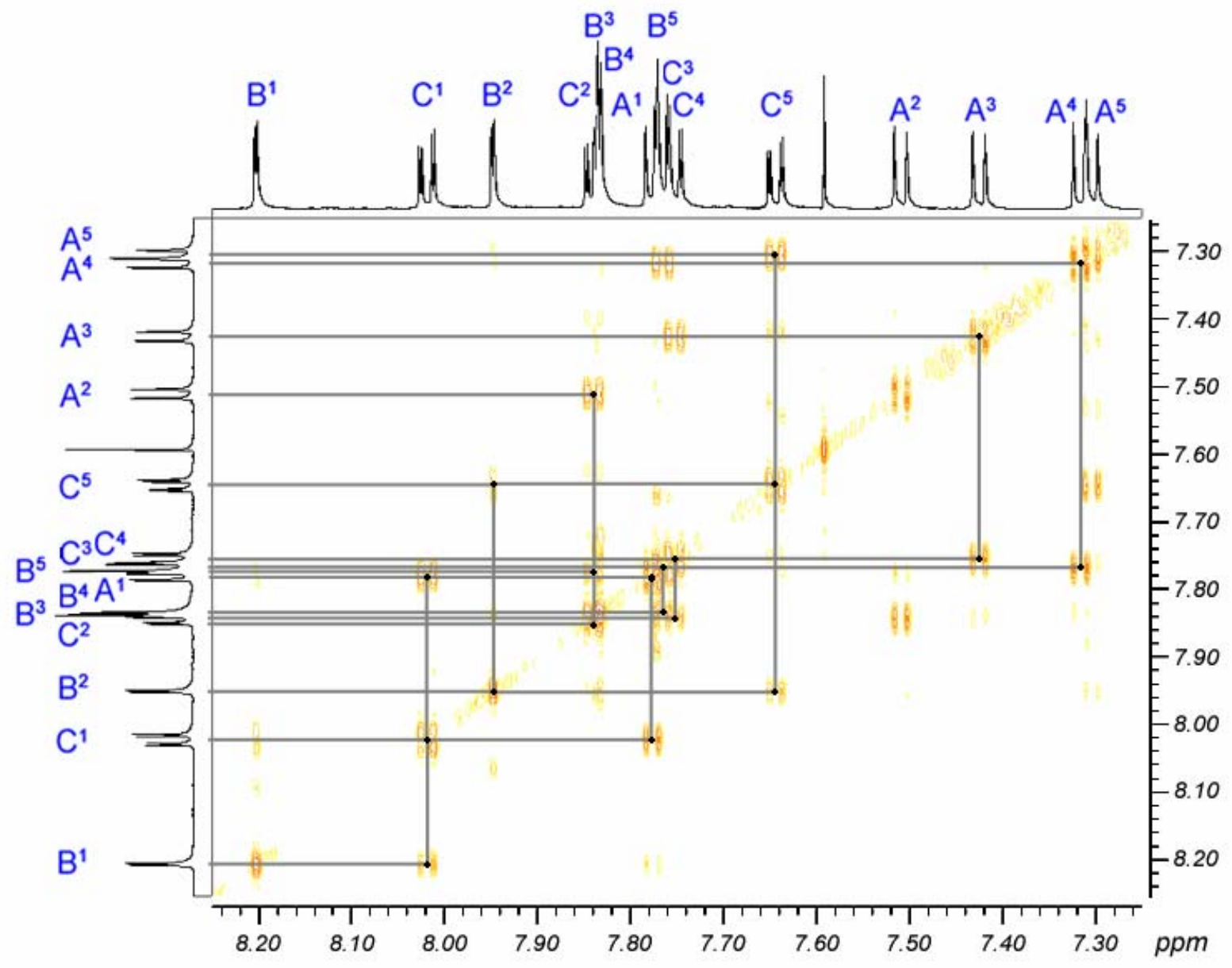

Figure S9. COSY spectrum of $\mathrm{Es}_{5}$, aromatic proton region. 


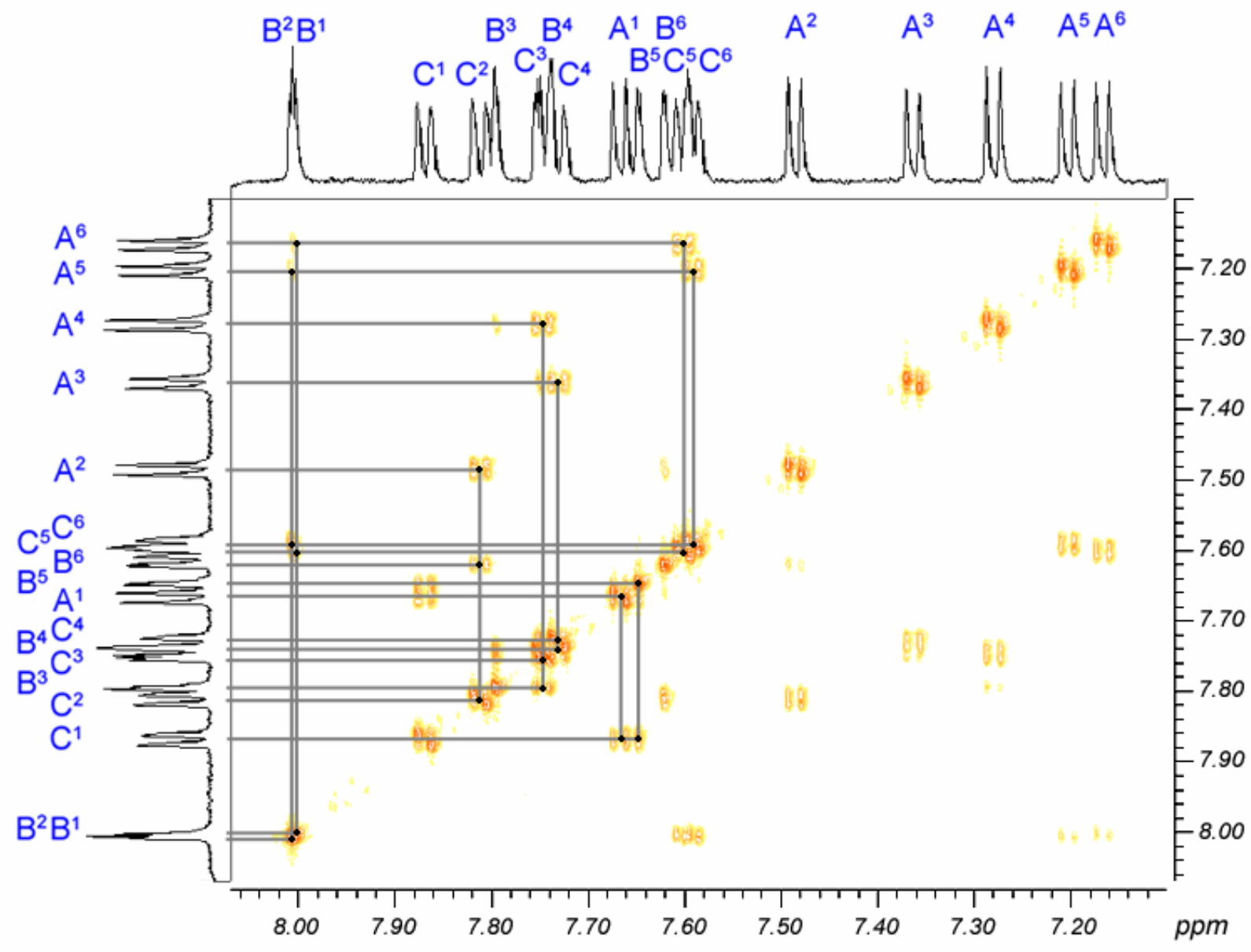

Figure S10. COSY spectrum of $\mathrm{Es}_{6}$, aromatic proton region. Slight shifting of ${ }^{1} \mathrm{H}$ peaks is apparent relative to that of the $\mathrm{Es}_{6} \mathrm{HMBC}$, as the $\mathrm{HMBC}$ was taken at $300 \mathrm{~K}$ and this COSY was taken at $305 \mathrm{~K}$. 


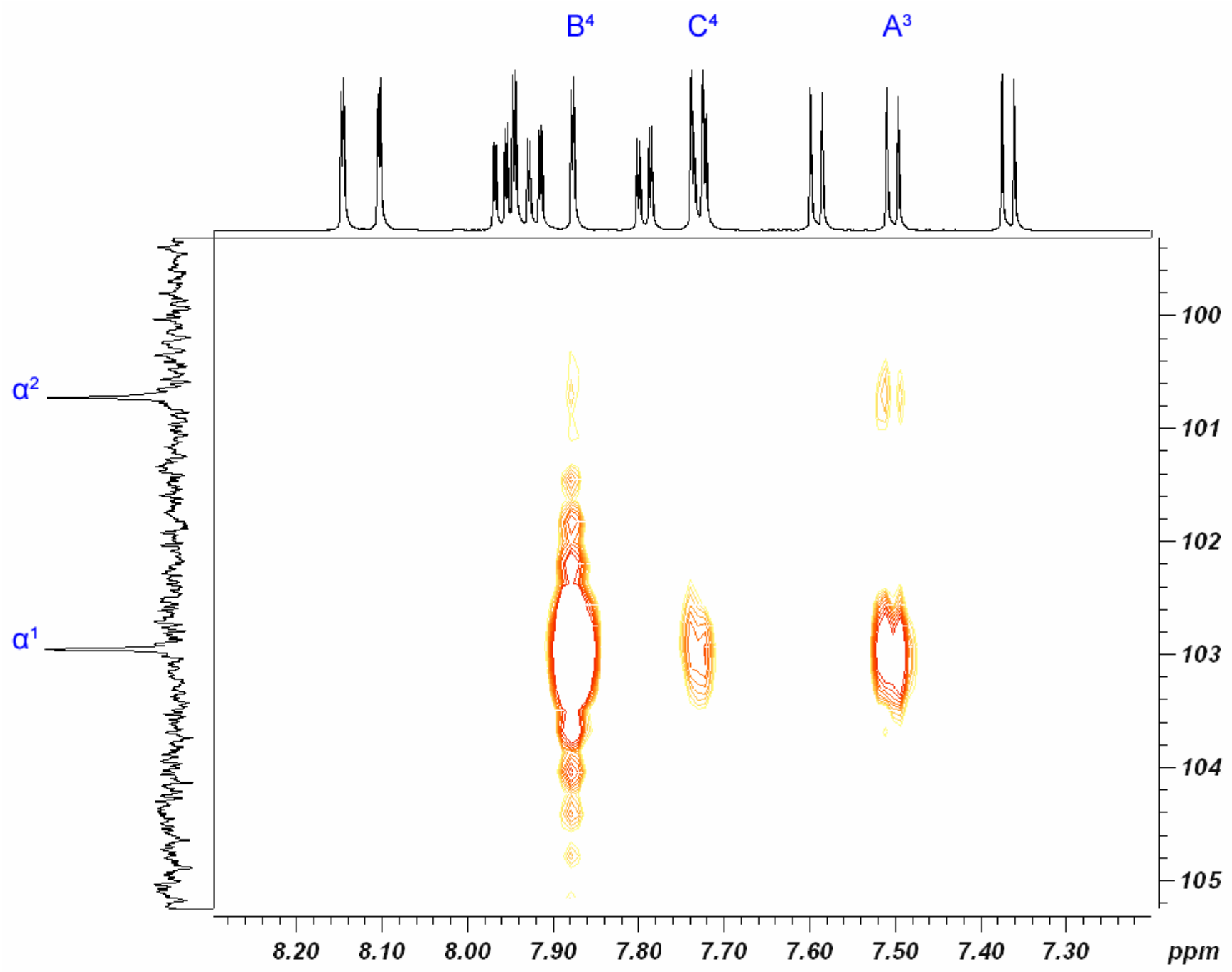

Figure S11. HMBC interactions between $\alpha^{1}$ and $\alpha^{2}$ and ring 1 protons of Es 4 . Same HMBC data set as previously mentioned. 


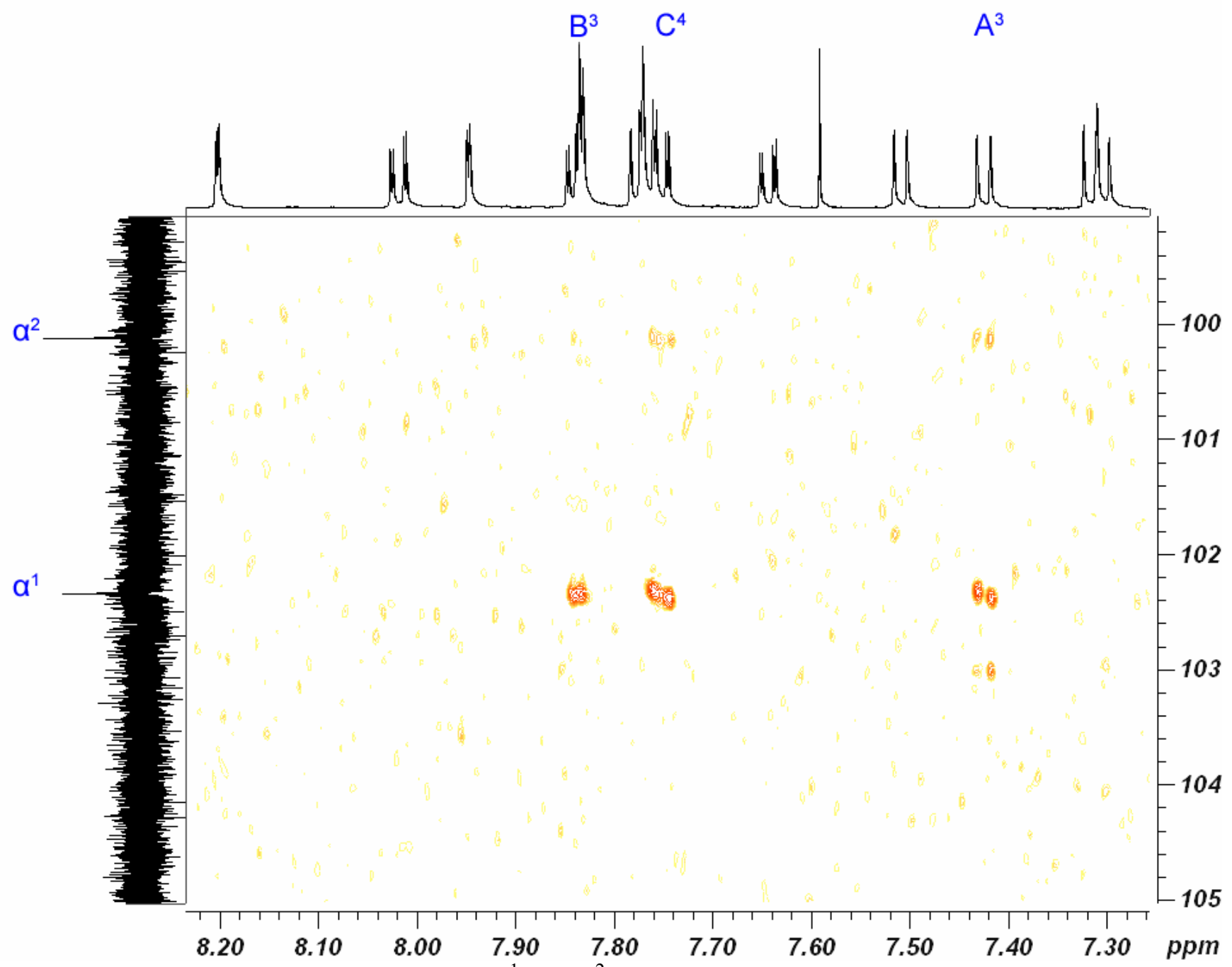

Figure S12. HMBC interactions between $\alpha^{1}$ and $\alpha^{2}$ and ring 1 protons of Es 5 . Same HMBC data set as previously mentioned. 


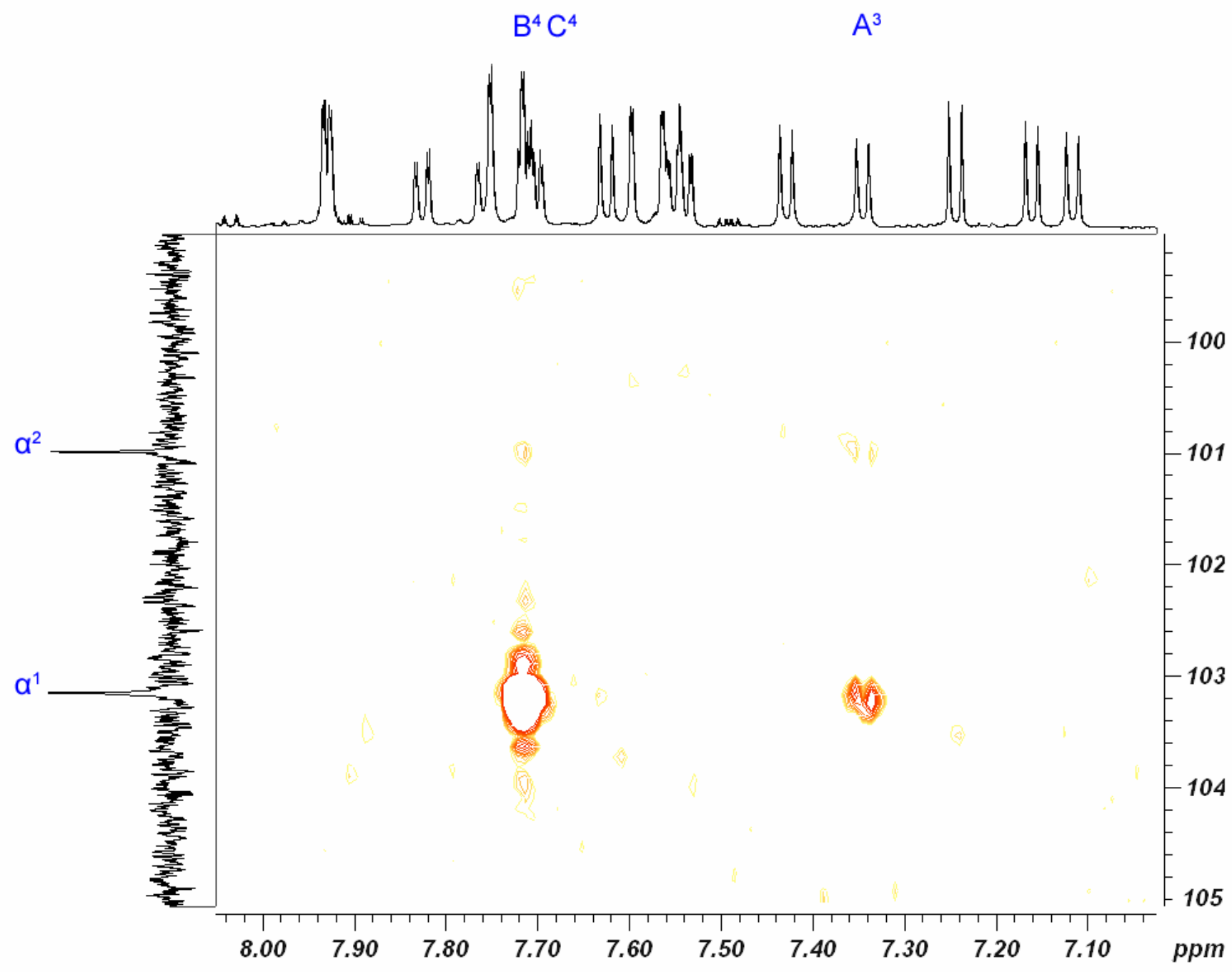

Figure S13. HMBC interactions between $\alpha^{1}$ and $\alpha^{2}$ and ring 1 protons of Es 6 . Same HMBC data set as previously mentioned. 

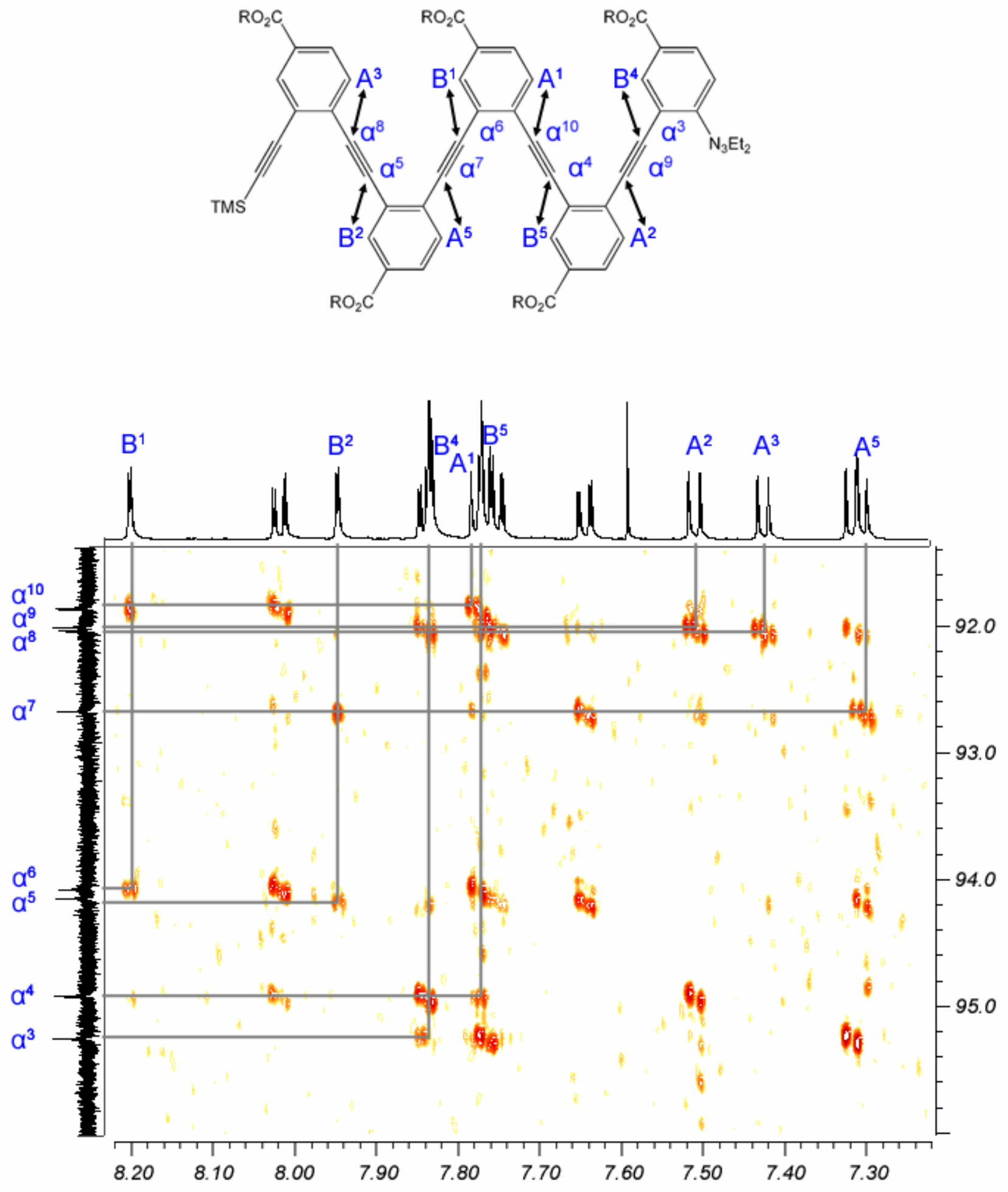

Figure S14. Short (crossing 2 carbon-carbon bonds) HMBC interactions between A and B protons of $\mathrm{Es}_{5}$ and acetylene carbons adjacent to each ring, shown in black arrows on the structure and connected by lines on the spectrum. Weaker, unlabeled crosspeaks indicate medium-range (crossing 3 carboncarbon bonds) $\mathrm{HMBC}$ interactions between $\mathrm{A}, \mathrm{B}$, and $\mathrm{C}$ protons of each ring and the adjacent acetylene carbons. 

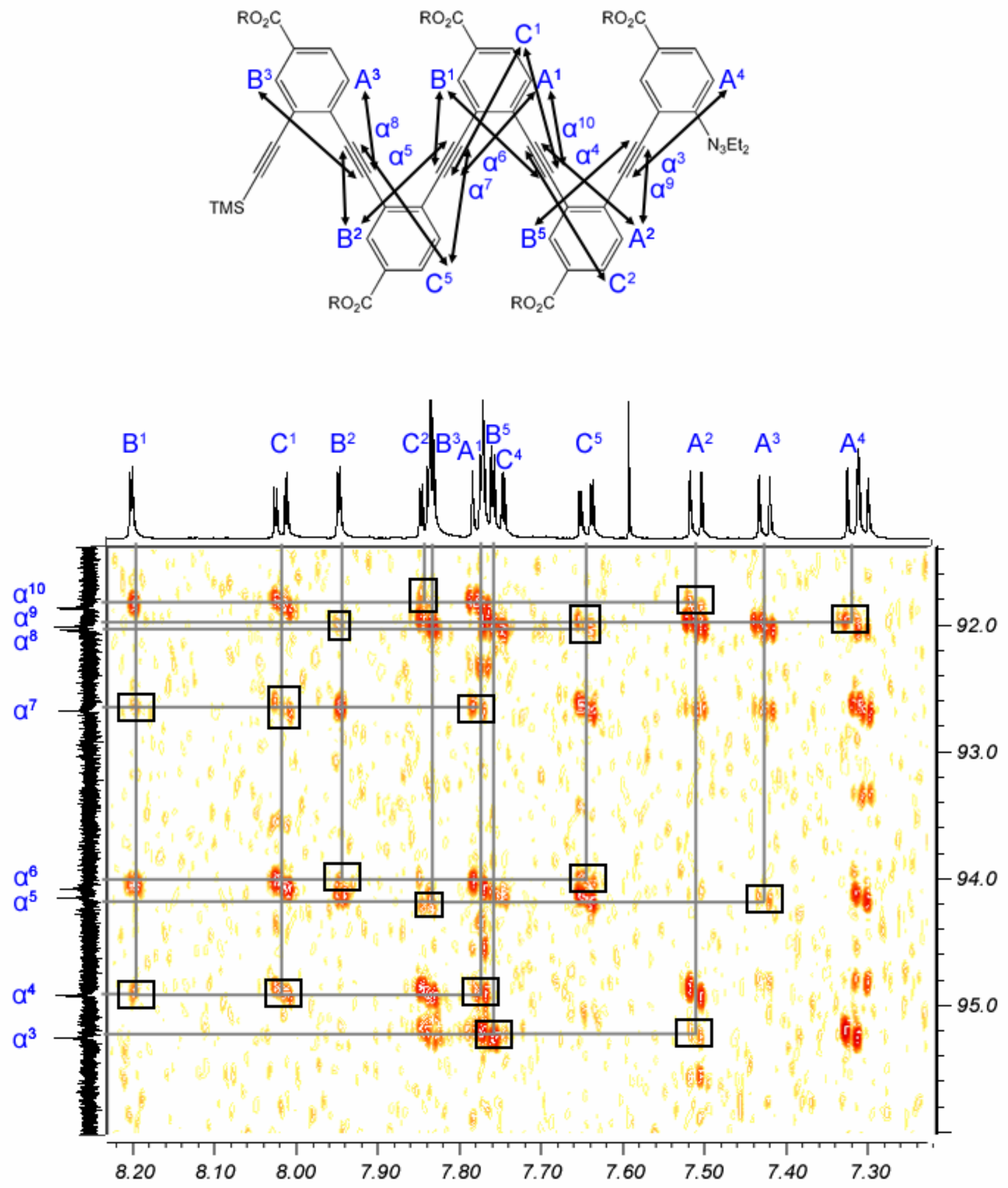

Figure S15. Long-range HMBC interactions across carbon-carbon triple bonds between aromatic protons of $E_{5}$ and acetylene carbons are shown in black arrows on the structure and connected by lines on the spectrum. Due to an NMR facility upgrade, the pulse program is optimized for longer-range couplings providing more inter-spin system signals than Figures 9 and 11. 

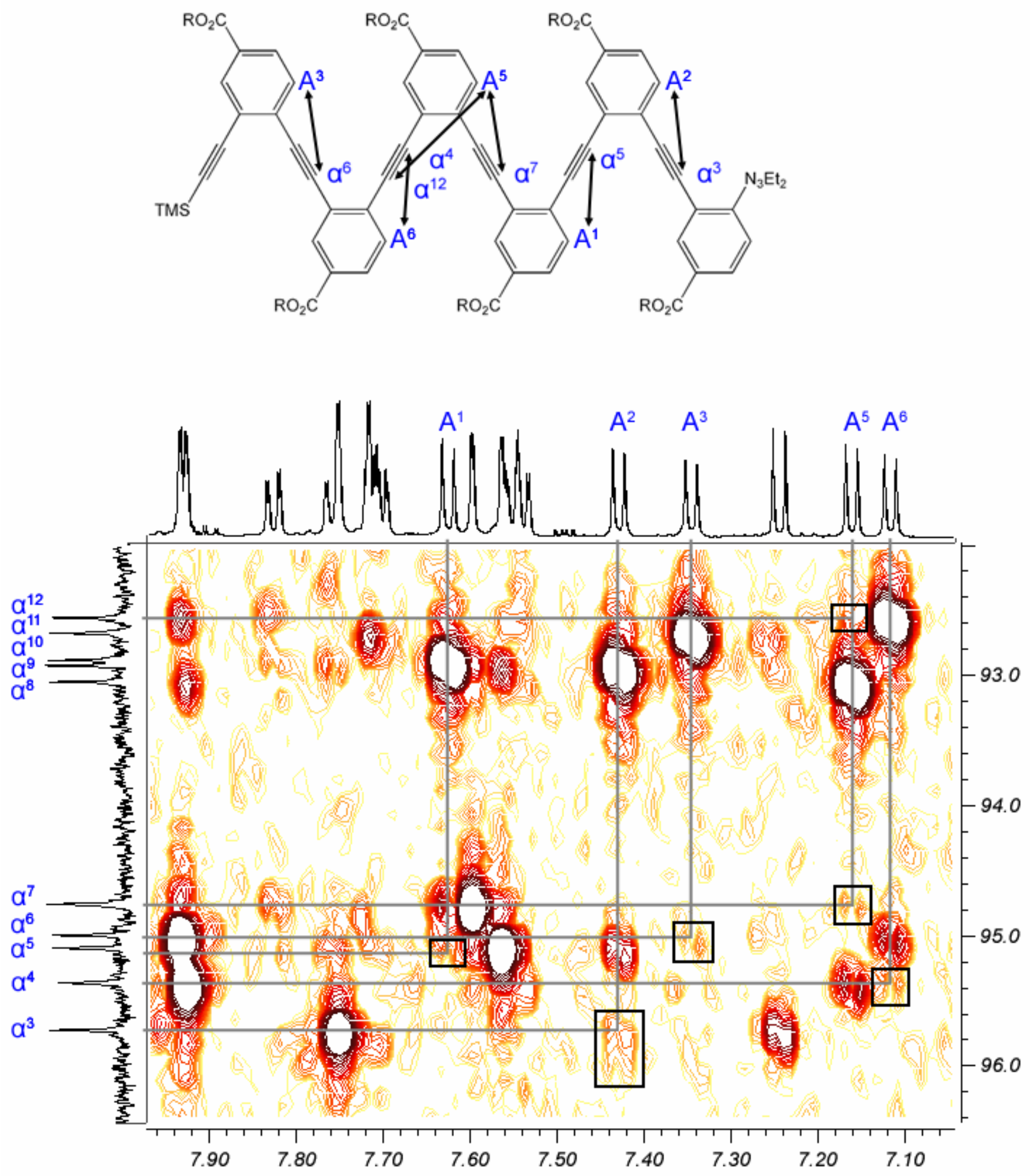

Figure S16. Long-range HMBC interactions across carbon-carbon triple bonds between aromatic protons of $\mathrm{Es}_{6}$ and acetylene carbons are shown in black arrows on the structure and connected by lines on the spectrum. Limited solubility of $\mathrm{Es}_{6}$ may have contributed to lower $\mathrm{S} / \mathrm{N}$ ratio such that fewer couplings were observed than in the spectra of $\mathrm{Es}_{4}$ and $\mathrm{Es}_{5}$. All the necessary signals for complete assignment are still present in this spectrum. 


\section{DETAILS AND DISCUSSION FOR ASSIGNMENT OF LONG-RANGE HMBC PEAKS}

Some of the weaker HMBC peaks are difficult to see in Figures S15-S16 referring to $\mathrm{Es}_{5}$ and $\mathrm{Es}_{6}$ respectively, mainly due to the need to set a consistent scale and zoom level. In this section, individual faint or overlapped long-range peaks are shown clearly in isolation in order to assist the reader.

Es5:

Although somewhat faint, $\alpha^{3}$ to $\mathrm{A}^{2}$ shows clear shape and splitting pattern.

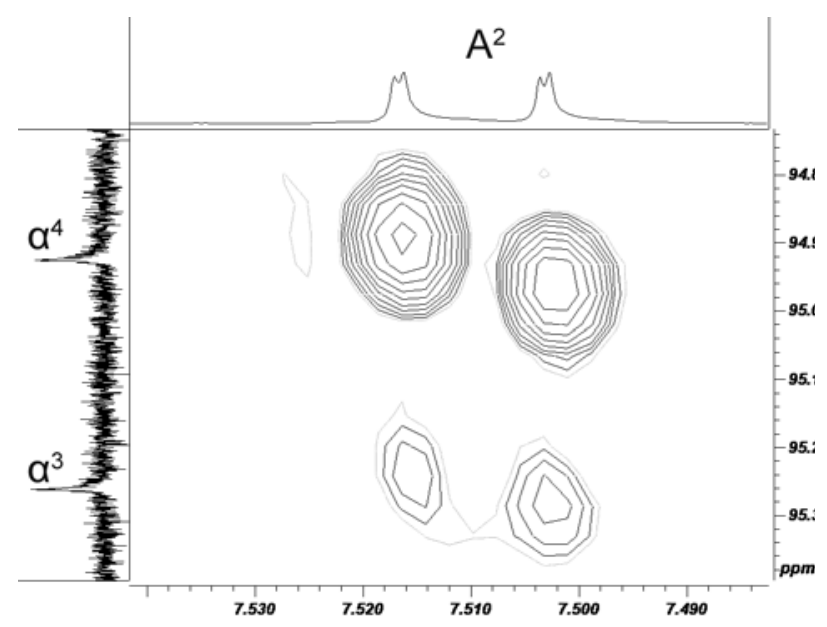

Although fairly faint, $\alpha^{8}$ to $\mathrm{B}^{2}$ and $\alpha^{7}$ to $\mathrm{B}^{1}$ (faint) may be clearly seen in the following graph wherein an appropriate contour level has been set to show those peaks and to exclude all noise. No marks or lines are present to obscure these peaks:

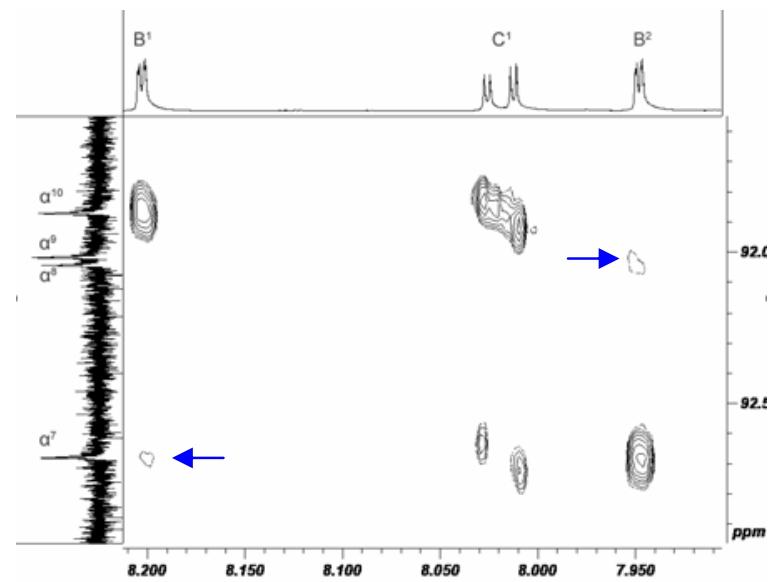


The $\alpha^{6}-\mathrm{B}^{2}$ peak is nearly a shoulder to the $\alpha^{5}-\mathrm{B}^{2}$ peak, but the peak certainly confirms an additional interaction. Shape compared to that of other B peaks as shown. Here the B interactions can be observed. $\alpha^{6}-\mathrm{B}^{1}$ is symmetric compared to $\alpha^{6}-\mathrm{B}^{2}$ since $\mathrm{B}^{2}$ has a strong interaction with $\alpha^{5}$ and a weak interaction with $\alpha^{6}$. $\mathrm{B}^{1}$ has no such weak interactions.

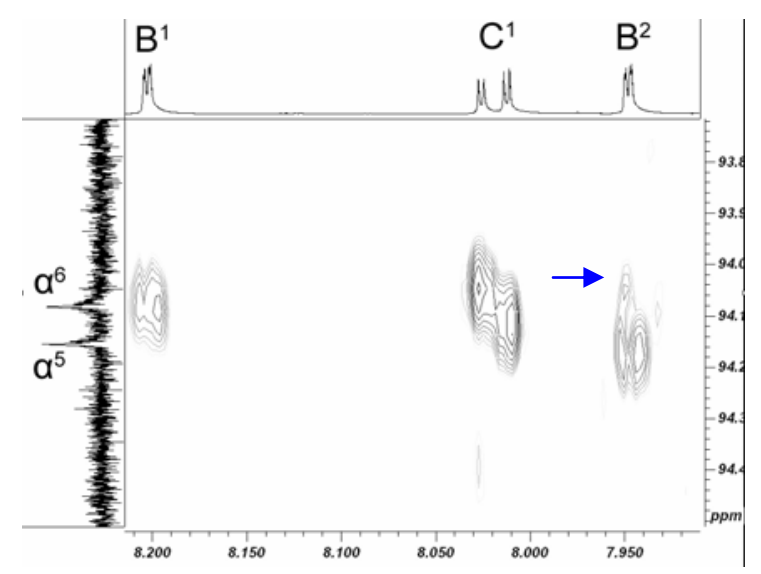

The $\alpha^{6}-C^{5}$ peak shows clear separation from the $\alpha^{5}-C^{5}$ peak.

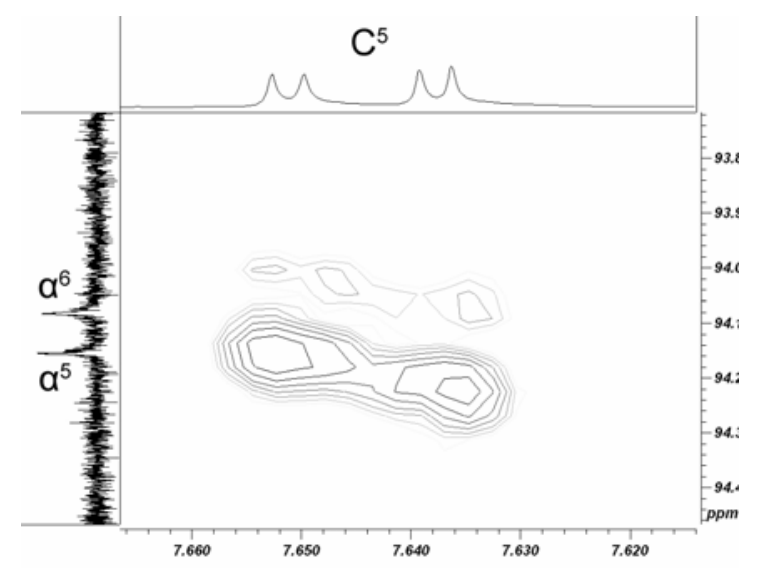

The $\alpha^{3}-B^{5}$ peak does overlap the $\alpha^{3}-C^{3}$ peak, but is visible by asymmetry - the $\alpha^{3}-C^{3}$ peak on its own would be symmetrical and have equal intensity on both maxima, similar to the $\alpha^{5}-C^{5}$ peak in the graph above. The narrow $\mathrm{B}$ peak resides on top of one of the $\mathrm{C}$ maxima and increases the total intensity of the left portion of $\mathrm{C}^{3}$.

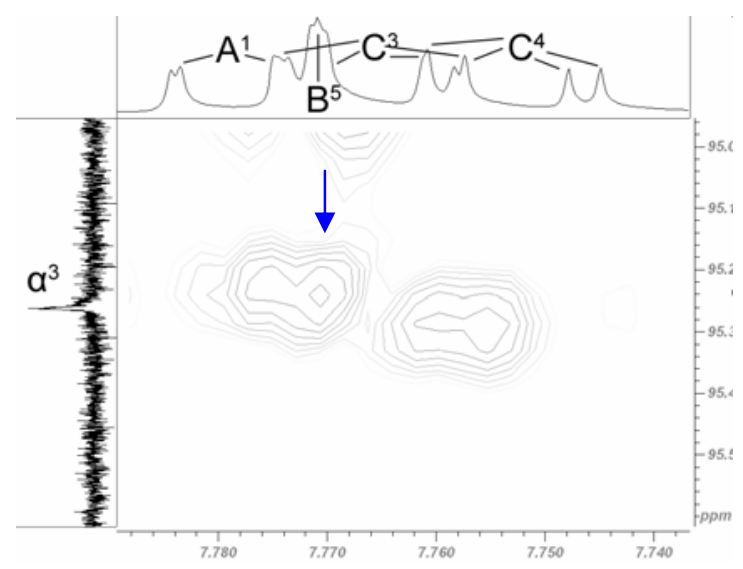


The $\alpha^{4}-A^{1}$ peak has a similar situation involving a wide A-type peak with 2 maxima, one of which is overlapped by the intense, but narrow $\alpha^{4}-B^{5}$ peak, and again the two maxima have different intensities which are readily apparent.

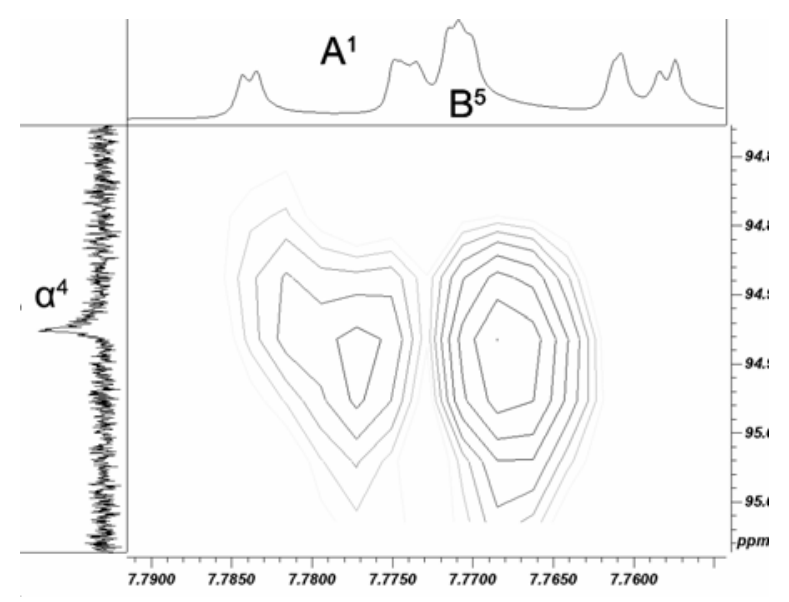

Some extraneous signals are seen in the spectrum of $\mathrm{Es}_{5}$ (e.g. $\alpha^{7}$ to $\mathrm{A}^{2}$ and $\mathrm{A}^{3}$ ). Most likely these are artifacts - the peakshapes for $\alpha^{7}$ to $\mathrm{A}^{2}$ and $\mathrm{A}^{3}$ do not match the splitting patterns of the ${ }^{1} \mathrm{H}$ spectrum above, but have an odd triplet appearance, compare to $\alpha^{7}-\mathrm{A}^{1}$ for example.

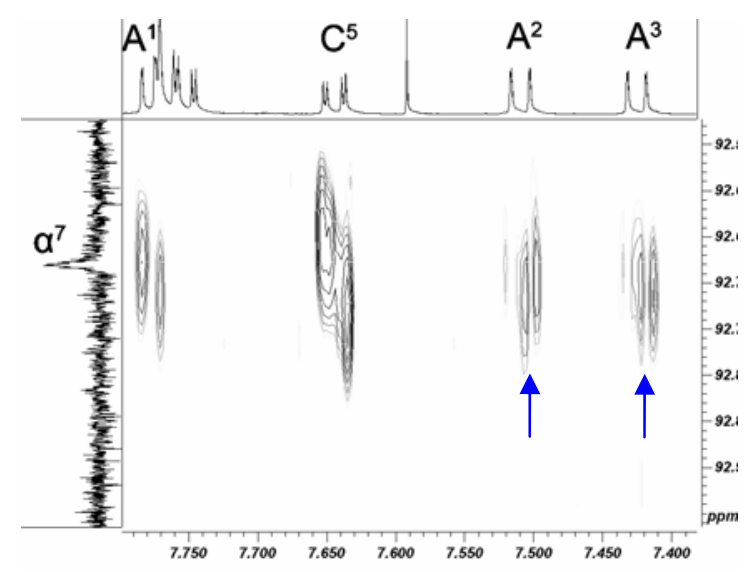

Even without the 7 peaks that have been discussed above, there remain sufficient HMBC interactions crossing triple bonds to make a full assignment of $\mathrm{Es}_{5}$. However, we believe that their use is reasonable and justified. 
Es6: plot.

The peaks for $\alpha^{12}$ to $A^{5}, \alpha^{5}$ to $A^{1}$, and $\alpha^{4}$ to $A^{6}$ are in somewhat congested regions of the HMBC

The $\alpha^{4}-A^{6}$ interaction shows clear separation from the nearby $\alpha^{4}-A^{5}$ and $\alpha^{6}-A^{6}$ peaks, as seen in the graph below where both maxima are easily distinct.

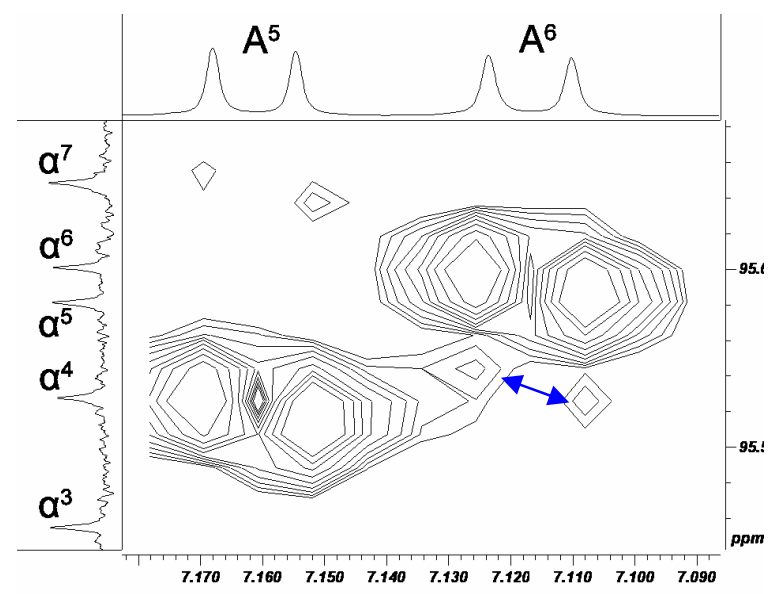

Admittedly the $\alpha^{12}-\mathrm{A}^{5}$ peak is especially weak, only present as a shoulder to the strong $\alpha^{8}-\mathrm{A}^{5}$ peak, as shown in the graph below. It is not of crucial importance to the assignment of $\mathrm{Es}_{6}$, as the same triple bond is crossed by the more clear $\alpha^{4}-\mathrm{A}^{6}$ interaction.

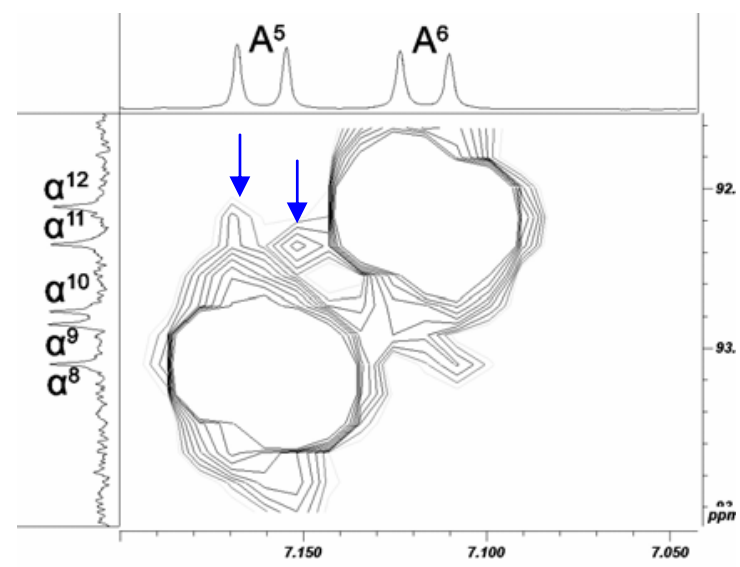


The $\alpha^{5}-\mathrm{A}^{1}$ peak is also a shoulder to the stronger $\alpha^{7}-\mathrm{A}^{1}$ peak, visible by observation of the lower fringe, as shown below.
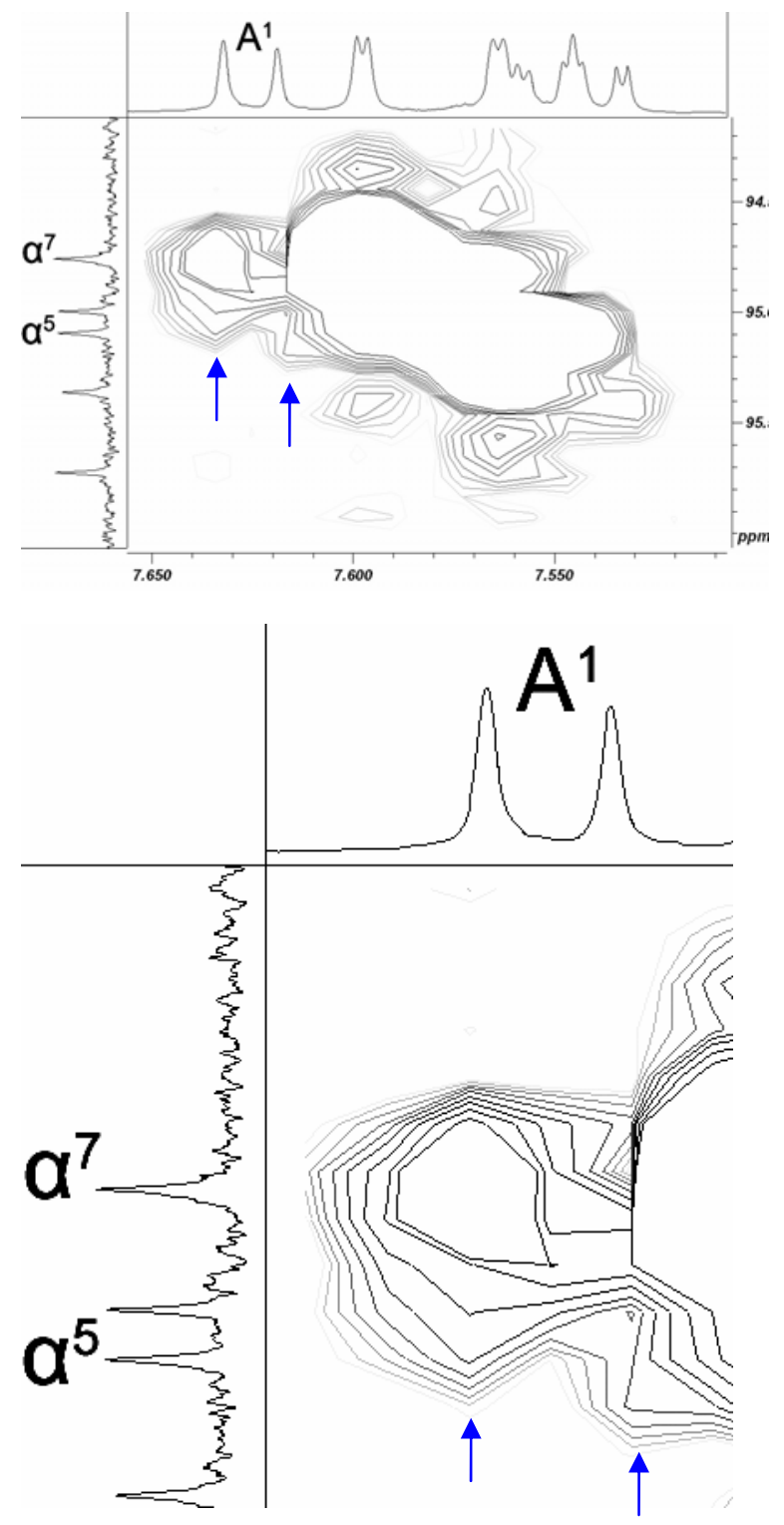

The $\alpha^{6}-\mathrm{A}^{3}, \alpha^{7}-\mathrm{A}^{5}$, and $\alpha^{3}-\mathrm{A}^{2}$ peaks, which connect rings 1-2, 3-4, and 5-6 respectively, are relatively easy to pick out of the plot shown in Figure S16. The discussion above regarding $\alpha^{4}-A^{6}$ should clarify that it is not obstructed by noise or overlapping peaks, but is also clear. As $\alpha^{4}-\mathrm{A}^{6}$ connects rings 2-3, these peaks give us a clear assignment for rings 1-4 and for rings 5-6, working from both ends inward as described in the manuscript. A connection between rings 4 and 5 is not necessary in order to make a complete assignment of Es $_{6}$.

This leaves the interactions $\alpha^{12}-\mathrm{A}^{5}$ and $\alpha^{5}-\mathrm{A}^{1}$, which provide connections between rings $2-3$ and 4-5 respectively. While $\alpha^{12}-\mathrm{A}^{5}$ is admittedly weak, it provides the same connectivity information as the $\alpha^{4}-\mathrm{A}^{6}$ interaction, which is unobstructed and easily visible above the noise floor. The $\alpha^{5}-\mathrm{A}^{1}$ interaction is overlapped by the stronger $\alpha^{7}-\mathrm{A}^{1}$, but is visible at the fringes and, in our view, should be included in our assignment of Es 6 . Without the use of the $\alpha^{5}-\mathrm{A}^{1}$ interaction, there is no connectivity between rings 4 and 5, but a complete and unambiguous assignment can still be made working from the ends inwards. However, we believe that the use of the $\alpha^{5}-\mathrm{A}^{1}$ to confirm our Es 6 assignment is reasonable and justified. 